\section{Cardiac ryanodine receptor calcium release deficiency syndrome}

\author{
Bo Sun ${ }^{1,2 *}$, Jinjing $\mathrm{Yao}^{1 *}$, Mingke $\mathrm{Ni}^{1}{ }^{*}$, Jinhong $\mathrm{Wei}^{1}$, Xiaowei Zhong ${ }^{1}$, Wenting Guo ${ }^{1}$, Lin Zhang ${ }^{1}$, \\ Ruiwu Wang ${ }^{1}$, Darrell Belke ${ }^{1}$, Yong-Xiang Chen ${ }^{1}$, Krystien V.V. Lieve ${ }^{3,4}$, Anders K. Broendberg ${ }^{5}$, \\ Thomas M. Roston ${ }^{6}$, Ivan Blankoff ${ }^{7}$, Janneke A. Kammeraad ${ }^{8}$, Johannes C. von Alvensleben ${ }^{9}$, \\ Julieta Lazarte $^{10}$, Alexander Vallmitjana ${ }^{11}$, Loryn J. Bohne ${ }^{12}$, Robert A. Rose ${ }^{12}$, Raul Benitez ${ }^{11}$, \\ Leif Hove-Madsen ${ }^{13}$, Carlo Napolitano ${ }^{4,14,15}$, Robert A. Hegele ${ }^{10}$, Michael Fill ${ }^{16}$, \\ Shubhayan Sanatani ${ }^{17 \dagger}$, Arthur A.M. Wilde ${ }^{3,4 \dagger}$, Jason D. Roberts ${ }^{18 \dagger}$, Silvia G. Priori ${ }^{4,14,15,19 \dagger}$, \\ Henrik K. Jensen ${ }^{5 t}$, S. R. Wayne Chen ${ }^{1,16 t}$
}

Cardiac ryanodine receptor (RyR2) gain-of-function mutations cause catecholaminergic polymorphic ventricular tachycardia, a condition characterized by prominent ventricular ectopy in response to catecholamine stress, which can be reproduced on exercise stress testing (EST). However, reports of sudden cardiac death (SCD) have emerged in EST-negative individuals who have loss-of-function (LOF) RyR2 mutations. The clinical relevance of RyR2 LOF mutations including their pathogenic mechanism, diagnosis, and treatment are all unknowns. Here, we performed clinical and genetic evaluations of individuals who suffered from SCD and harbored an LOF RyR2 mutation. We carried out electrophysiological studies using a programed electrical stimulation protocol consisting of a long-burst, long-pause, and short-coupled (LBLPS) ventricular extra-stimulus. Linkage analysis of RyR2 LOF mutations in six families revealed a combined logarithm of the odds ratio for linkage score of 11.479 for a condition associated with SCD with negative EST. A RyR2 LOF mouse model exhibited no catecholamine-provoked ventricular arrhythmias as in humans but did have substantial cardiac electrophysiological remodeling and an increased propensity for early afterdepolarizations. The LBLPS pacing protocol reliably induced ventricular arrhythmias in mice and humans having RyR2 LOF mutations, whose phenotype is otherwise concealed before SCD. Furthermore, treatment with quinidine and flecainide abolished LBLPS-induced ventricular arrhythmias in model mice. Thus, RyR2 LOF mutations underlie a previously unknown disease entity characterized by SCD with normal EST that we have termed RyR2 $\mathrm{Ca}^{2+}$ release deficiency syndrome (CRDS). Our study provides insights into the mechanism of CRDS, reports a specific CRDS diagnostic test, and identifies potentially efficacious anti-CRDS therapies.

\section{INTRODUCTION}

The cardiac ryanodine receptor (RyR2) governs the release of $\mathrm{Ca}^{2+}$ from the sarcoplasmic reticulum (SR), which is essential for excitationcontraction coupling in the heart and has a major role in the pathogenesis of cardiac arrhythmias (1-4). Mutations in RYR2 cause catecholaminergic polymorphic ventricular tachycardia (CPVT), a cardiac channelopathy associated with malignant ventricular arrhythmias (VAs) and sudden cardiac death (SCD) (4-8). CPVT develops due to RyR2 gain-of-function (GOF) mutations and the resultant diastolic spontaneous $\mathrm{Ca}^{2+}$ release and delayed afterdepolarizations
(DADs) that can trigger malignant polymorphic ventricular tachycardia (VT). The hallmark of CPVT is increasing ventricular ectopy that progresses to more complex arrhythmias, most typically bidirectional VT, in response to catecholamine stress (4). CPVT can be reliably reproduced on exercise stress testing (EST), making EST a useful CPVT diagnostic tool (4-11).

In contrast to CPVT-causing RyR2 GOF mutations, recent work has begun to identify mutations among survivors of cardiac arrest that exhibit a RyR2 loss of function (LOF) or suppression of function on in vitro functional testing (12-16). However, the clinical

\footnotetext{
${ }^{1}$ Libin Cardiovascular Institute of Alberta, Department of Physiology and Pharmacology, University of Calgary, Calgary, AB T2N 4Z6, Canada. ${ }^{2}$ Medical School, Kunming University of Science and Technology, Kunming 650504, China. ${ }^{3}$ Amsterdam University Medical Centre, location AMC, Heart Center, Department of Clinical and Experimental Cardiology, Amsterdam 1105AZ, Netherlands. ${ }^{4}$ European Reference Network 'ERN GUARD-Heart', Amsterdam, Netherlands. ${ }^{5}$ Department of Cardiology, Aarhus University Hospital, and Department of Clinical Medicine, Health, Aarhus University, Palle Juul-Jensens Blv 99, DK-8200 Aarhus N, Denmark. ${ }^{6}$ Division of Cardiology, Department of Medicine, University of British Columbia, Vancouver, BC V5Z 1M9, Canada. ${ }^{7}$ C.H.U. Charleroi, Hôpital Civil Marie Curie Chaussée de Bruxelles 1406042 Charleroi, Belgium. ${ }^{8}$ Department of Pediatric Cardiology, Sophia Children's Hospital, Erasmus University Medical Centre, Doctor Molewaterplein 40, 3015 GD Rotterdam, Netherlands. ${ }^{9}$ Division of Cardiology, Heart Institute, Children's Hospital Colorado, University of Colorado, Aurora, CO 80045, USA. ${ }^{10}$ Department of Medicine and Robarts Research Institute, Schulich School of Medicine and Dentistry, Western University, London, ON N6A 5B7, Canada. ${ }^{11}$ Department of Automatic Control, Universitat Politècnica de Catalunya, 08034 Barcelona, Spain. ${ }^{12}$ Departments of Cardiac Sciences and Physiology and Pharmacology, Libin Cardiovascular Institute of Alberta, Cumming School of Medicine, University of Calgary, Calgary, AB T2N 4Z6, Canada. ${ }^{13}$ Biomedical Research Institute Barcelona (IIBB-CSIC) and IIB Sant Pau, Hospital de Sant Pau, Barcelona 08025, Spain. ${ }^{14}$ Division of Cardiology and Molecular Cardiology, IRCCS Maugeri Foundation-University of Pavia, 27100 Pavia, Italy. ${ }^{15}$ Department of Molecular Medicine, University of Pavia, 27100 Pavia, Italy. ${ }^{16}$ Department of Physiology and Biophysics, Rush University Medical Center, Chicago, IL 60612, USA. ${ }^{17}$ Child and Family Research Institute, Department of Pediatrics, University of British Columbia, Vancouver, BC V6H 3V4, Canada. ${ }^{18}$ Section of Cardiac Electrophysiology, Division of Cardiology, Department of Medicine, Western University, London, ON N6A 5A5, Canada. ${ }^{19}$ Molecular Cardiology Laboratory, Centro de Investigaciones Cardiovasculares Carlos III, 28029 Madrid, Spain.

*These authors contributed equally to this work.

†Corresponding author. Email: swchen@ucalgary.ca (S.R.W.C.); hkjensen@clin.au.dk (H.K.J.); silvia.priori@icsmaugeri.it (S.G.P.); jason.roberts@lhsc.on.ca (J.D.R.); a.a.wilde@amsterdamumc.nl (A.A.M.W.); ssanatani@cw.bc.ca (S.S.)
} 
ramifications of RyR2 LOF mutations are unclear. Patients having RyR2 LOF mutations do not exhibit the CPVT phenotype. The phenotype of carriers of RyR2 LOF mutations, aside from their fatal or near-fatal cardiac events, has been otherwise within normal limits on cardiac testing $(12,15-17)$. As a result, these patients are often assigned a cryptic diagnosis of idiopathic ventricular fibrillation.

Clarification of the clinical relevance of RyR2 LOF mutations has become imperative. It is currently unknown whether these genetic variants are truly causal for a potentially life-threatening disorder or merely incidental and noncausal genomic background noise, which is notably high in the RYR2 gene $(\sim 3 \%)(18,19)$. Should these genetic variants be drivers of malignant arrhythmias, this would be critical knowledge for SCD risk management of the patients and their family members. Here, we provide clinical, genetic, and functional evidence that definitively establishes RyR2 LOF mutations as being causative for a previously unknown disease entity that we call RyR2 $\mathrm{Ca}^{2+}$ release deficiency syndrome (CRDS). We also introduce a diagnostic testing maneuver for this otherwise concealed condition and provide evidence for medical therapies that may effectively prevent life-threatening events in patients having RyR2 LOF mutations.

\section{RESULTS}

\section{A previously unknown cardiac arrhythmia linked to RyR2 LOF}

We identified six families in whom the probands had suffered SCD or aborted SCD (aSCD) and had rare RyR2 mutations (Q3774L, I3995V, D4112N, T4196I, D4646A, or Q4879H) that were subsequently identified to result in LOF or suppression of function on in vitro functional evaluation (Fig. 1, fig. S1, and Table 1). None of the probands or genotype-positive family members exhibited a typical CPVT phenotype. Combining these six families (with 20 SCD/ aSCD) and four previously reported families (with 11 SCD/aSCD) having RyR2 LOF mutations (I4855M, A4860G, S4938F, and K4594R/ I2075T) $(12,15-17)$ showed that 31 of $46(67 \%)$ RyR2 mutant carriers experienced SCD/aSCD, but none of the 46 (0\%) RyR2 mutationnegative individuals suffered from life-threatening VAs (fig. S1 and Table 1).

To statistically evaluate the link between RyR2 LOF mutations and this arrhythmic disease entity, we used the two-point linkage analysis using LIPED (20). This analysis revealed a combined maximal logarithm of the odds ratio for linkage (LOD) score from six families (I3995V, D4112N, T4196I, D4646A, I4855M, and $\mathrm{R} 4594 \mathrm{~K} / \mathrm{I} 2075 \mathrm{~T}$ ) of 11.479 at $0 \%$ recombination, which is equivalent to odds of $301,300,000,000: 1$ in favor of complete linkage between phenotype and genotype (table S1). There were too few informative meioses in three families (Q3774L, A4860G, and $\mathrm{S} 4938 \mathrm{~F}$ ) to calculate linkage. $\mathrm{Q} 4879 \mathrm{H}$ is a de novo aSCDassociated RyR2 mutation found in the proband, whereas neither parent was clinically or genetically affected (see the "Linkage analysis in RYR2 mutation families" section in the Supplementary Materials).

The RyR2 mutations identified are located in the RyR2's central domain, U-motif, S2-S3 loop, pore domain (S5-S6), or C-terminal domain-all of which are critical for channel gating (Fig. 2A). To assess the functional impact of these RyR2 mutations, we measured caffeine-induced $\mathrm{Ca}^{2+}$ release and store overload-induced $\mathrm{Ca}^{2+}$ release
(SOICR) in human embryonic kidney (HEK) 293 cells expressing either RyR2 wild type (WT) or mutants. RyR2 mutations Q3774L, I3995V, D4112N, T4196I, D4646A, Q4879H, I4855M, A4860G, and $\mathrm{S} 4938 \mathrm{~F}$ markedly suppressed caffeine-induced $\mathrm{Ca}^{2+}$ release and SOICR without altering the store capacity. Specifically, higher caffeine concentrations and higher intra-ER (endoplasmic reticulum) $\mathrm{Ca}^{2+}$ concentrations were required to activate the mutant RyR2s, compared to RyR2-WT (Fig. 2, B to D, and figs. S2 and S3). The expression of these RyR2 mutants was comparable to that of the RyR2-WT (fig. S3, A and B). Some mutants had additional actions on SOICR termination threshold and/or RyR2-mediated fractional $\mathrm{Ca}^{2+}$ release (fig. S3, C to F). Furthermore, all six newly identified RyR2 mutations impaired the cytosolic $\mathrm{Ca}^{2+}$ activation and diminished the luminal $\mathrm{Ca}^{2+}$ activation of single RyR2 channels (Fig. 2, $\mathrm{E}$ and F, and fig. S4). As for the RyR2 K4594R/I2075T double mutation, it also markedly suppressed caffeine-induced $\mathrm{Ca}^{2+}$ release largely due to the impact of the K4594R mutation, because the impact of the double RyR2 K4594R/I2075 mutation and the single K4594R mutation is similar (fig. S2D). Note that, although all of these RyR2 LOF mutants exhibited reduced channel activities, they were still functional (i.e., not dead). Collectively, our clinical, genetic, functional, and statistical analyses definitively establish the link between RyR2 LOF and a previously unknown disease entity characterized by SCD/aSCD with negative EST. We call this previously unknown disease entity RyR2-CRDS.

\section{RyR2-D4646A ${ }^{+/-}$diminishes spontaneous $\mathrm{SR} \mathrm{Ca}^{2+}$ release and stress-induced VAs}

To explore the mechanism by which CRDS RyR2 mutations cause SCD, we generated a knock-in mouse model expressing one of the RyR2 LOF mutations, D4646A (fig. S5). As in humans, only heterozygous RyR2-D4646A mutant mice (RyR2-D4646A ${ }^{+/-}$), but no homozygous mutant mice, were produced, suggesting that homozygous RyR2-D4646A $\mathrm{A}^{+/+}$mutation is embryonically lethal. We first determined the impact of the heterozygous RyR2-D4646A ${ }^{+/-}$mutation on intracellular $\mathrm{Ca}^{2+}$ handling. Specifically, we assessed the propensity for store overload-induced spontaneous $\mathrm{SR} \mathrm{Ca}^{2+}$ release, a well-known consequence of typical CPVT RyR2 mutations $(4,21,22)$, in WT and D4646 $\mathrm{A}^{+/-}$mutant intact hearts. Elevating extracellular $\mathrm{Ca}^{2+}$ concentration to 4 to $8 \mathrm{mM}$ (to promote $\mathrm{SR} \mathrm{Ca}^{2+}$ overload) triggered spontaneous $\mathrm{Ca}^{2+}$ waves in WT hearts. The same maneuver induced little or no $\mathrm{Ca}^{2+}$ waves in the $\mathrm{D}^{2} 646 \mathrm{~A}^{+/-}$mutant hearts (Fig. 3, A to C). The D4646 $\mathrm{A}^{+/-}$mutant hearts were also resilient to caffeine- and epinephrine-promoted spontaneous $\mathrm{Ca}^{2+}$ waves (fig. S6, A to C). Thus, this CRDS RyR2 LOF mutation markedly suppresses spontaneous $\mathrm{Ca}^{2+}$ waves in intact hearts, unlike the wellstudied CPVT-linked RyR2 GOF mutations (4, 21-24).

We also tested the susceptibility of the RyR2-D4646A ${ }^{+/-}$mutant mice to stress-induced VAs. High doses of caffeine and epinephrine are commonly used as pharmacological promoters in CPVT model mouse studies (23). However, the same pharmacological mixture did not promote VAs in the D4646A ${ }^{+/-}$mice (Fig. 3, D to G). Again, unlike typical CPVT RyR2 GOF mutations, this CRDS RyR2 LOF mutation protected the heart against stress-induced VAs. This action is consistent with the negative EST and the absence of typical CPVT phenotypes in human RyR2 D4646A ${ }^{+/-}$mutant carriers (see the "Genetic analyses and clinical evaluation of RyR2 mutant carriers presenting with life-threatening VAs distinct from CPVT" section in the Supplementary Materials). 


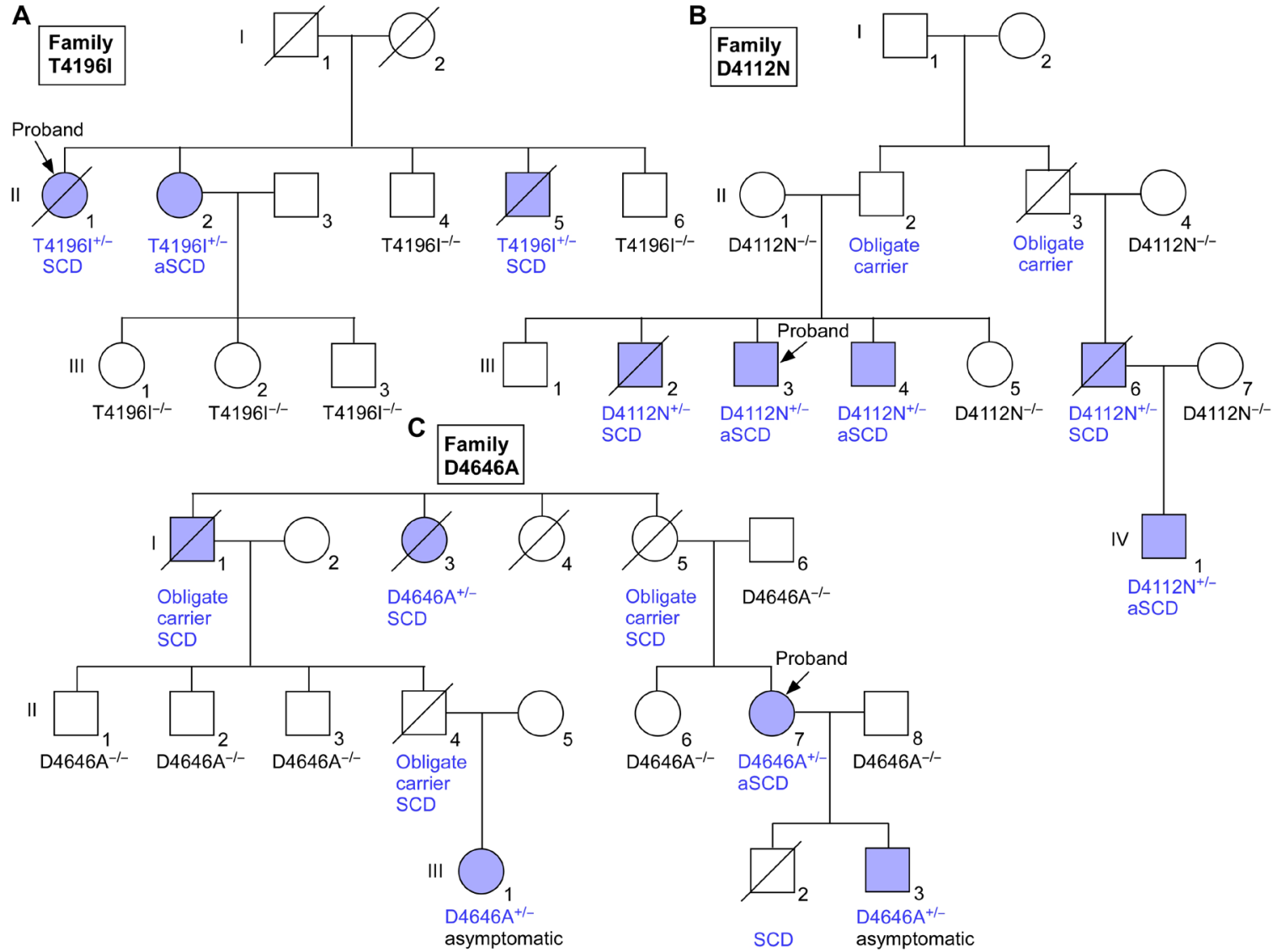

D

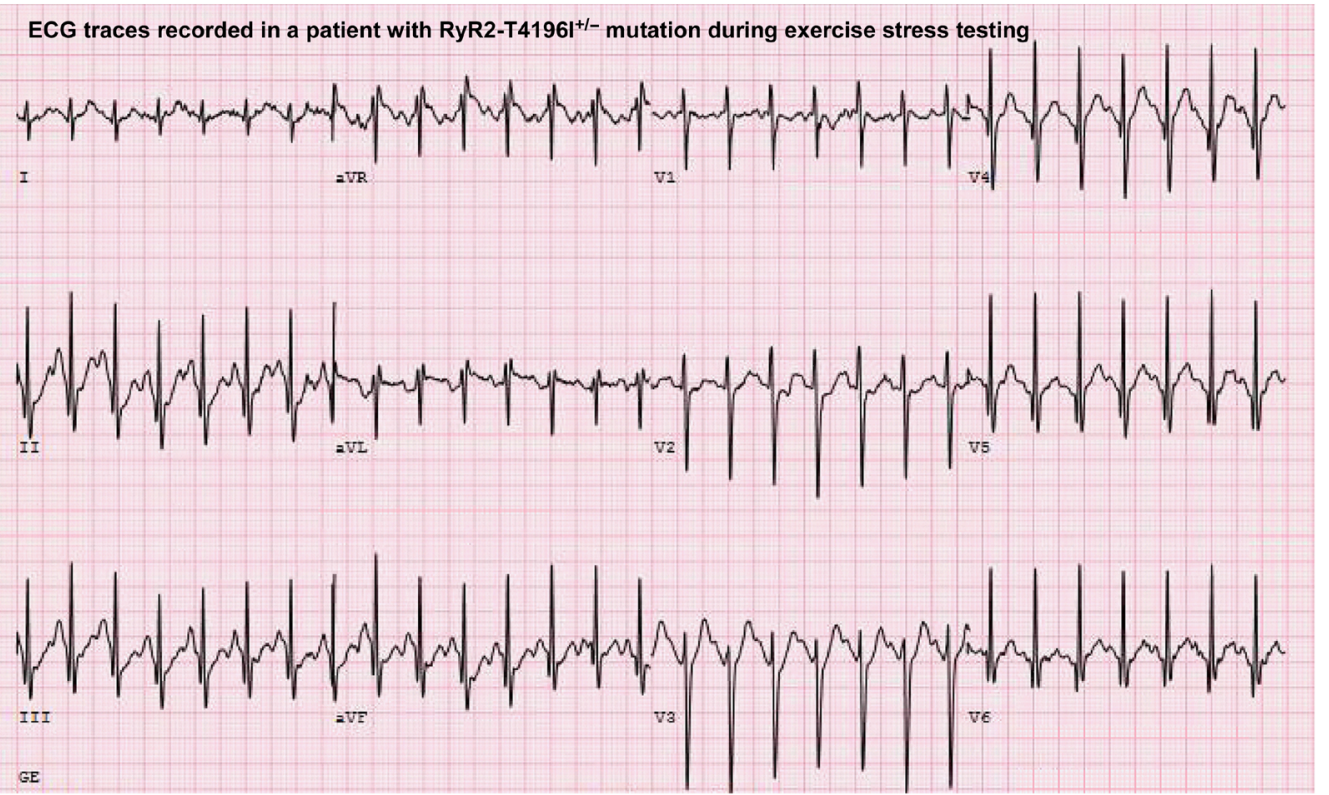

Fig. 1. Pedigrees of families with non-CPVT-associated RyR2 mutations. Families with RyR2 mutations T4196I ${ }^{+/-}$(A), D4112N $\mathrm{N}^{+/-}$(B), and D4646A ${ }^{+/-}$(C). (D) ECG traces recorded during exercise stress testing. The letters on the ECG traces annotate the leads. There were no PVCs observed during the entire testing, and QT intervals under all conditions tested were within the normal limit. Squares and circles indicate males and females, respectively; blue symbols represent mutation carriers/obligate carriers; open symbols represent mutation-negative/unaffected persons; symbols with a slash represent deceased persons. Probands are indicated by an arrow. SCD, sudden cardiac death; aSCD, aborted SCD. 
Table 1. Primary symptoms and exercise stress test outcome of RyR2 mutant carriers presenting with ventricular arrhythmias distinct from CPVT. SCD, sudden cardiac death; aSCD, aborted SCD; SUD, sudden unexplained death; LVNC, left ventricular non-compaction cardiomyopathy; IVF, idiopathic ventricular fibrillation; scTdP, short-coupled variant of Torsade de Pointes; LOF, loss of function. RyR2 mutations I4855M, A4860G, S4938F, and K4594R/I2075T have been previously reported (12,15-17). The functional impact of RyR2-14855M, RyR2-A4860G, and RyR2-S4938F has been previously reported (13-16), but that of the K4594R/I2075T was characterized in this study.

\begin{tabular}{|c|c|c|c|c|c|}
\hline RyR2 mutation & Primary symptoms & $\begin{array}{l}\text { Exercise stress test } \\
\text { (EST) }\end{array}$ & $\begin{array}{l}\text { Symptomatic mutant } \\
\text { carriers (total) }\end{array}$ & $\begin{array}{c}\text { Symptomatic } \\
\text { mutant-negative } \\
\text { carriers (total) }\end{array}$ & Mutation impact \\
\hline Q3774L $^{+/-}$ & aSCD & Negative & $1(4)$ & $0(1)$ & LOF \\
\hline $\mathrm{D}^{1112 \mathrm{~N}^{+/-}}$ & SCD/aSCD & Negative & $5(7)$ & $0(4)$ & LOF \\
\hline $\mathrm{T} 196 \mathrm{I}^{+/-}$ & $\mathrm{SCD} / \mathrm{aSCD}$ & Negative & $3(3)$ & $0(5)$ & LOF \\
\hline $14855 \mathrm{M}^{+/-}$ & SCD/LVNC & Negative & $2(3)$ & $0(3)$ & LOF \\
\hline $\mathrm{A} 4860 \mathrm{G}^{+/-}$ & SCD/IVF & Negative & $2(3)$ & $0(1)$ & LOF \\
\hline $\mathrm{S}_{4} 938 \mathrm{~F}^{+--}$ & scTdP & Negative & $1(3)$ & $0(4)$ & LOF \\
\hline $\mathrm{K} 594 \mathrm{R}^{+/-} / 12075 \mathrm{~T}^{+/-}$ & $\mathrm{SCD} / \mathrm{aSCD}$ & Negative & $6(7)$ & $0(4)$ & LOF \\
\hline
\end{tabular}

\section{RyR2-D4646A ${ }^{+/-}$promotes $\mathrm{Ca}^{2+}$ alternans, prolongs $\mathrm{Ca}^{2+}$ release refractoriness, alters action potential waveform, and enhances EAD propensity}

To determine whether the RyR2 D4646 $\mathrm{A}^{+/-}$mutation affects depolarization-induced SR $\mathrm{Ca}^{2+}$ release, we measured $\mathrm{Ca}^{2+}$ transients evoked by electrical stimulation at different frequencies in intact hearts. Although the $\mathrm{D} 4646 \mathrm{~A}^{+/-}$mutation suppresses spontaneous SR $\mathrm{Ca}^{2+}$ release, the amplitude, time to peak, and decay time of depolarization-induced $\mathrm{Ca}^{2+}$ transients at low stimulation frequencies in CRDS D4646 $\mathrm{A}^{+/-}$mutant hearts or isolated cardiomyocytes were similar to those in WT (Fig. 3, H to M, and fig. S6, D to I). At high stimulation frequencies, however, mutant hearts were more prone to $\mathrm{Ca}^{2+}$ alternans (fig. S7, A to D). When stimulated at $10 \mathrm{~Hz}$, WT hearts showed little or no $\mathrm{Ca}^{2+}$ alternans, whereas mutant hearts displayed substantial $\mathrm{Ca}^{2+}$ alternans. The effect of the CRDS D $4646 \mathrm{~A}^{+-}$ mutation on $\mathrm{SR} \mathrm{Ca}^{2+}$ release refractoriness was also measured, and the D4646 $\mathrm{A}^{+/-}$mutation prolonged the refractoriness (fig. S7, E to G).

To determine whether this CRDS RyR2 mutation alters action potential (AP) properties, the whole-cell patch-clamp technique was used to record APs in isolated WT and D4646 $\mathrm{A}^{+/}$mutant ventricular myocytes. The AP waveform in the $\mathrm{D} 4646 \mathrm{~A}^{+/-}$cells was markedly different from that in WT cells. The AP duration at $50 \%\left(\mathrm{APD}_{50}\right)$ was shorter, and $\mathrm{APD}_{90}$ was prolonged in CRDS $\mathrm{D} 4646 \mathrm{~A}^{+/-}$mutant ventricular myocytes, whereas the AP amplitude and resting membrane potential were unchanged (Fig. 3, N to R). Intriguingly, D4646 $\mathrm{A}^{+/-} \mathrm{mu}-$ tant cells were highly susceptible to early afterdepolarizations (EADs) compared to WT cells (Fig. 3, S and T). Thus, this CRDS RyR2 LOF mutation $\mathrm{D} 646 \mathrm{~A}^{+/-}$produces systolic arrhythmogenic abnormalities, in stark contrast to the diastolic arrhythmogenic $\mathrm{Ca}^{2+}$ dysregulation associated with CPVT-causing RyR2 GOF mutations.

\section{RyR2-D4646A ${ }^{+/-}$causes cardiac electrophysiological and structural remodeling}

Although the RyR2-D4646A ${ }^{+/-}$mutation suppresses RyR2 function, the amplitude of $\mathrm{Ca}^{2+}$ transients in mutant ventricular myocytes was unchanged. This implies that the suppression of RyR2 function may have been compensated for by an increase in surface $\mathrm{Ca}^{2+}$ influx. To test this possibility, we measured the L-type $\mathrm{Ca}^{2+}$ channel current $\left(I_{\mathrm{CaL}}\right)$ using whole-cell patch-clamp. The $I_{\mathrm{CaL}}$ current density in $\mathrm{D}_{4646 \mathrm{~A}^{+-}}$mutant ventricular myocytes was markedly enhanced compared to WT (Fig. 4, A to C). If more $\mathrm{Ca}^{2+}$ enters the cell per AP, then one would expect more to exit as well. Consistent with this prediction, the $\mathrm{Na} / \mathrm{Ca}$ exchange current $\left(I_{\mathrm{NCX}}\right)$ was substantially augmented in $\mathrm{D} 4646 \mathrm{~A}^{+/-}$mutant ventricular myocytes (Fig. 4, D to G). This increased $I_{\mathrm{CaL}}$ and $I_{\mathrm{NCX}}$ current explains the prolonged $\mathrm{APD}_{90}$ and the higher EAD propensity in the mutant cells.

To explore why the $\mathrm{D}_{4} 646 \mathrm{~A}^{+/-}$mutation shortens $\mathrm{APD}_{50}$, we measured the transient outward $\mathrm{K}^{+}$current $\left(I_{\mathrm{to}}\right)$ and the Na current $\left(I_{\mathrm{Na}}\right)$. These are currents that govern early repolarization. The $I_{\mathrm{to}}$ current density and voltage-dependent activation were markedly enhanced in RyR2-D4646A ${ }^{+/-}$mutant ventricular myocytes compared to WT (Fig. 4, H to K, and fig. S8, A to D). As for $I_{\mathrm{Na}}$, RyR2$\mathrm{D}_{4} 646 \mathrm{~A}^{+/-}$ventricular myocytes have a leftward (hyperpolarization) shift in voltage-dependent activation and inactivation compared to WT (Fig. 4, L to P). Together, these data indicate that the CRDS RyR2 D4646A $\mathrm{A}^{+/-}$mutation results in a substantial electrophysiological remodeling of surface membrane currents.

We next performed histological and echocardiographic analyses to assess whether the $\mathrm{D} 4646 \mathrm{~A}^{+/-}$mutation affects cardiac structure and mechanical function. There were no marked differences in cardiac morphology and fibrosis between the WT and D4646 $\mathrm{A}^{+/-}$mutant hearts as revealed by hematoxylin/eosin and picrosirius red staining (fig. S8, E and F). The D4646A ${ }^{+/-}$hearts displayed slightly reduced heart rate $(\sim 10 \%)$ and moderately increased $(\sim 20 \%)$ left ventricular wall thickness, whereas other echocardiographic parameters (such as ejection fraction, fractional shortening, stroke volume, and cardiac output) were unchanged (table S2).

To gain insights into the molecular basis of this electrophysiological and structural remodeling, we performed immunoblotting and MitoSOX staining analyses. The surface expression of Cav1.2 
A

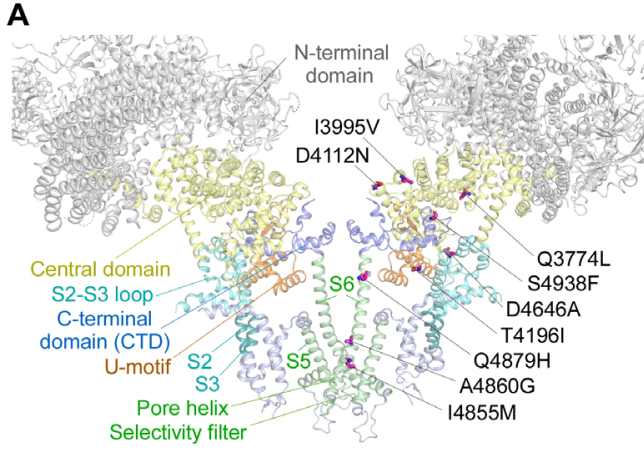

C
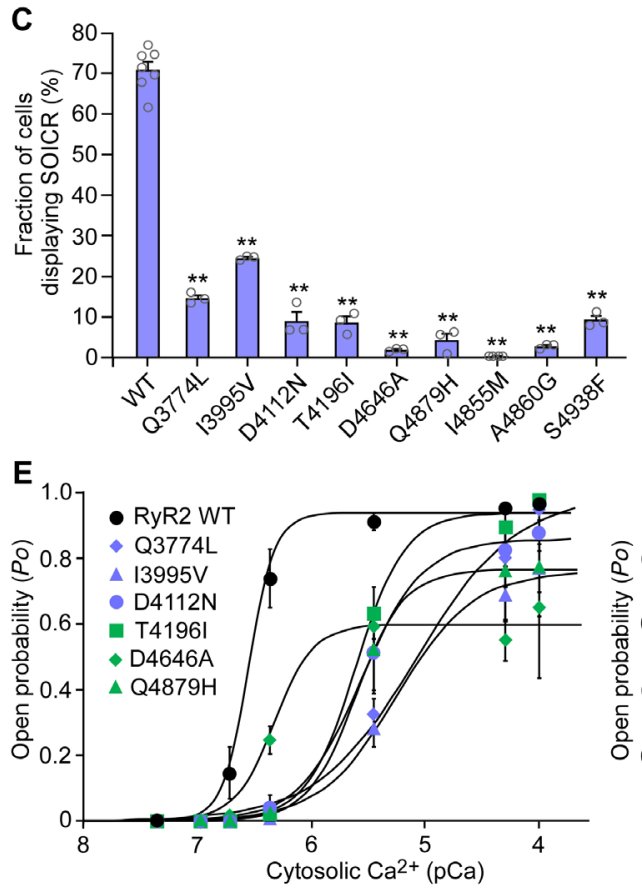
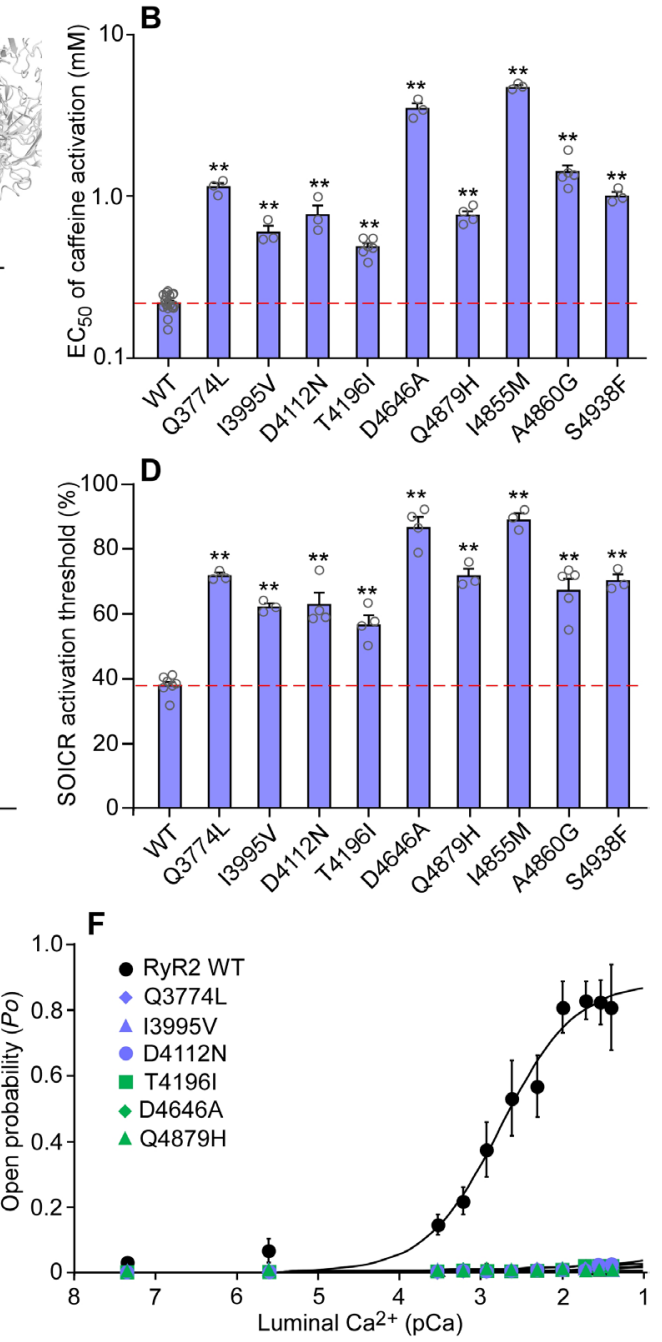

Fig. 2. Functional characterization of non-CPVT-associated RyR2 mutations. (A) Three-dimensional localization of RyR2 mutations. (B) $\mathrm{EC}_{50}$ (median effective concentration) values of caffeine-induced $\mathrm{Ca}^{2+}$ releases in $\mathrm{HEK} 293$ cells. Data are means \pm SEM from RyR2-WT $(n=18)$, Q3774L $(n=3)$, I3995V $(n=3), \mathrm{D} 4112 \mathrm{~N}(n=3)$, T4196I $(n=6), \mathrm{D} 4646 \mathrm{~A}$ $(n=3), \mathrm{Q} 4879 \mathrm{H}(n=4), 14855 \mathrm{M}(n=3), \mathrm{A} 4860 \mathrm{G}(n=5)$, and S4938F $(n=3)$ (one-way ANOVA with Dunnett's post hoc test, ${ }^{* *} P<0.01$ versus WT). Red dashed line represents the WT level. (C) Percentages of HEK293 cells displaying Ca ${ }^{2+}$ oscillations at $2 \mathrm{mM}$ extracellular $\mathrm{Ca}^{2+}\left(\left[\mathrm{Ca}^{2+}\right]_{0}\right)$. Data are means \pm SEM from RyR2-WT $(n=7), \mathrm{Q} 3776 \mathrm{~L}(n=3), 13995 \mathrm{~V}$ $(n=3), \mathrm{D} 4112 \mathrm{~N}(n=3), \mathrm{T} 4196 \mathrm{I}(n=3), \mathrm{D} 4646 \mathrm{~A}(n=3), \mathrm{Q} 4879 \mathrm{H}(n=3), 14855 \mathrm{M}(n=3), \mathrm{A} 4860 \mathrm{G}(n=3)$, and S4938F $(n=3)$ (one-way ANOVA with Dunnett's post hoc test, ${ }^{* *} P<0.01$ versus WT). (D) Activation thresholds for store overload-induced $\mathrm{Ca}^{2+}$ release (SOICR) in HEK293 cells. Data are means \pm SEM from RyR2-WT $(n=7), \mathrm{Q} 3774 \mathrm{~L}(n=3)$, I3995V ( $n=3), \mathrm{D} 4112 \mathrm{~N}(n=4), \mathrm{T} 4196 \mathrm{I}(n=4), \mathrm{D} 4646 \mathrm{~A}(n=4), \mathrm{Q} 4879 \mathrm{H}(n=3), 14855 \mathrm{M}(n=3), \mathrm{A} 4860 \mathrm{G}(n=5)$, and S4938F $(n=3)$ (one-way ANOVA with Dunnett's post hoc test, ${ }^{* *} P<0.01$ versus WT) ( $n$ refers to the number of separate experiments). Red dashed line represents the WT level. (E) Open probability (Po) of single RyR2-WT or mutant channels activated by cytosolic $\mathrm{Ca}^{2+}$. Data are means \pm SEM from RyR2-WT ( $n=6$ channels), Q3774L $(n=5), 13995 \mathrm{~V}(n=5)$, D4112N ( $n=5)$, T4196I $(n=5), \mathrm{D} 4646 \mathrm{~A}(n=6)$, and Q4879H $(n=5)$. (F) Po of single RyR2-WT or mutant channels activated by luminal $\mathrm{Ca}^{2+}$. Data are means \pm SEM from RyR2-WT ( $n=8$ channels), Q3774L $(n=5), 13995 \mathrm{~V}(n=5), \mathrm{D} 4112 \mathrm{~N}$ $(n=5), \mathrm{T} 4196 \mathrm{I}(n=5), \mathrm{D} 4646 \mathrm{~A}(n=5)$, and Q4879H $(n=5)$.

was unchanged (fig. S8, G to J). MitoSOX staining revealed no evidence for altered oxidative stress in $\mathrm{D}_{4} 646 \mathrm{~A}^{+/-}$ventricular myocytes (fig. S8, K and L). There were no significant differences in the total protein expression of Cav1.2, Cavß2, NCX1.1, Nav1.5, Kv4.2, Kv4.3, KChIP2, KChIP4, RyR2, SERCA2a, CASQ2, triadin, or junctin between WT and D4646A ${ }^{+-}$hearts (figs. S9 and S10). There was an increase in the expression of the phosphatase PP2 (protein phosphatase 2) in the $\mathrm{D} 646 \mathrm{~A}^{+/-}$heart compared to WT, whereas

phosphorylation of RyR2 (at S2808 and S2814) and calcium/calmodulindependent protein kinase II (CaMKII) and the total protein expression of CaM, PP1, protein kinase A (PKA), and CaMKII were unchanged (fig. S10). Thus, alterations in the expression of channel modulators (e.g., PP2) may in part contribute to the remodeling.

\section{A diagnostic test for RyR2-CRDS}

There are currently no clinical tests to distinguish potentially lethal RyR2 LOF mutations from presumably benign RyR2 rare variants, observed in $\sim 3 \%$ of the general population $(18,19)$. To address this unmet need, we leveraged our RyR2-D4646 $\mathrm{A}^{+/-}$mouse model to develop a RyR2-CRDS-specific diagnostic procedure. We performed in vivo intracardiac electrogram recordings to assess the VA inducibility of three common stimulation protocols: a burst pacing, a long pause, and a short-coupled premature ventricular complex (S1S2 stimulation). None of the common stimulation protocols reliably induced VAs in CRDS RyR2-D4646A ${ }^{+/-}$mice (Fig. 5, A to F). We then identified an effective three-component protocol. This protocol consists of a long-burst, long-pause, and short-coupled (LBLPS) extra-stimulus. This LBLPS protocol robustly and consistently triggered VAs in CRDS RyR2-D4646A ${ }^{+/-}$mutant mice (Fig. 5, G to L). To assess CRDS specificity of this LBLPS stimulation protocol, we applied it to both WT and CPVT RyR2-R4496C ${ }^{+/-}$GOF mutant mice. The LBLPS stimulation protocol induced little or no VAs in WT or CPVT mice (Fig. 5, G to L), indicating that the LBLPS protocol is RyR2-CRDS specific.

An electrical pattern reminiscent of the LBLPS protocol was captured just before VA onset by an implantable cardioverter defibrillator (ICD) in a patient harboring the CRDS RyR2-D4112N ${ }^{+/-}$ mutation (Fig. 5M). This observation and the robust effectiveness of LBLPS in mice prompted us to study the LBLPS protocol in humans harboring CRDS RyR2 mutations. We successfully recruited and tested two patients with RyR2-CRDS harboring the $\mathrm{I} 3995 \mathrm{~V}^{+/-}$and $\mathrm{T}_{4} 196 \mathrm{I}^{+/-}$mutation, respectively. The LBLPS stimulation sequence induced an episode of polymorphic VA in these patients with CRDS RyR2 (Fig. 5, N and O). Although still in the proof-ofconcept stage in humans, LBLPS appears to be a viable CRDSspecific diagnostic tool. This is critical given that the phenotype of 
A Pacing

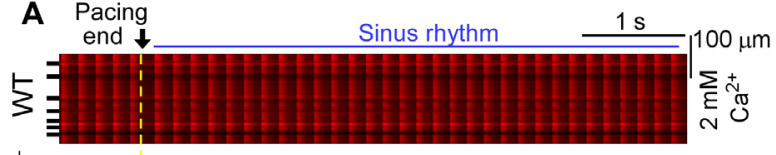

+
+
0
0
0
0
0

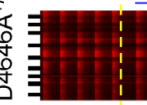

B

3

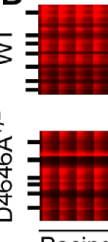

$\overline{\text { Pacing }}$

c

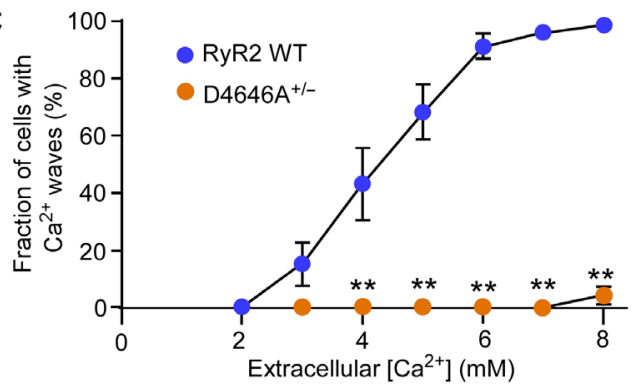

Spontaneous $\mathrm{Ca}^{2+}$ release
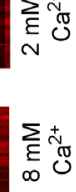

D WT mice

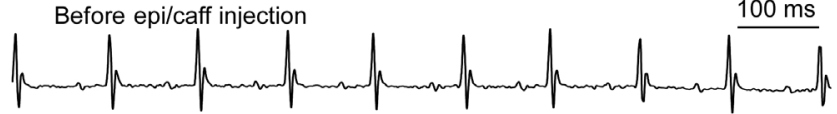

After epi/caff (3.0 mg/150 mg, high dose) injection

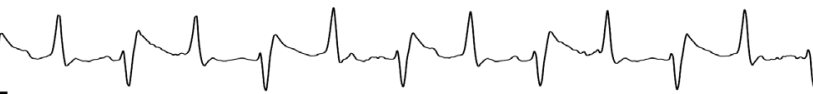

E

D4646A ${ }^{+/-}$mutant mice

Before epi/caff injection

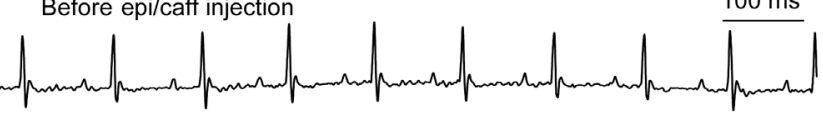

After epi/caff (3.0 mg/150 mg, high dose) injection
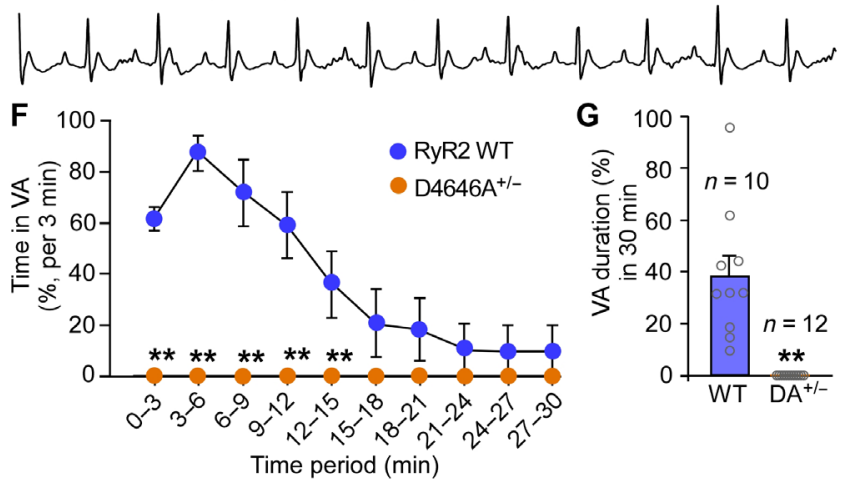

Time period $(\mathrm{min})$
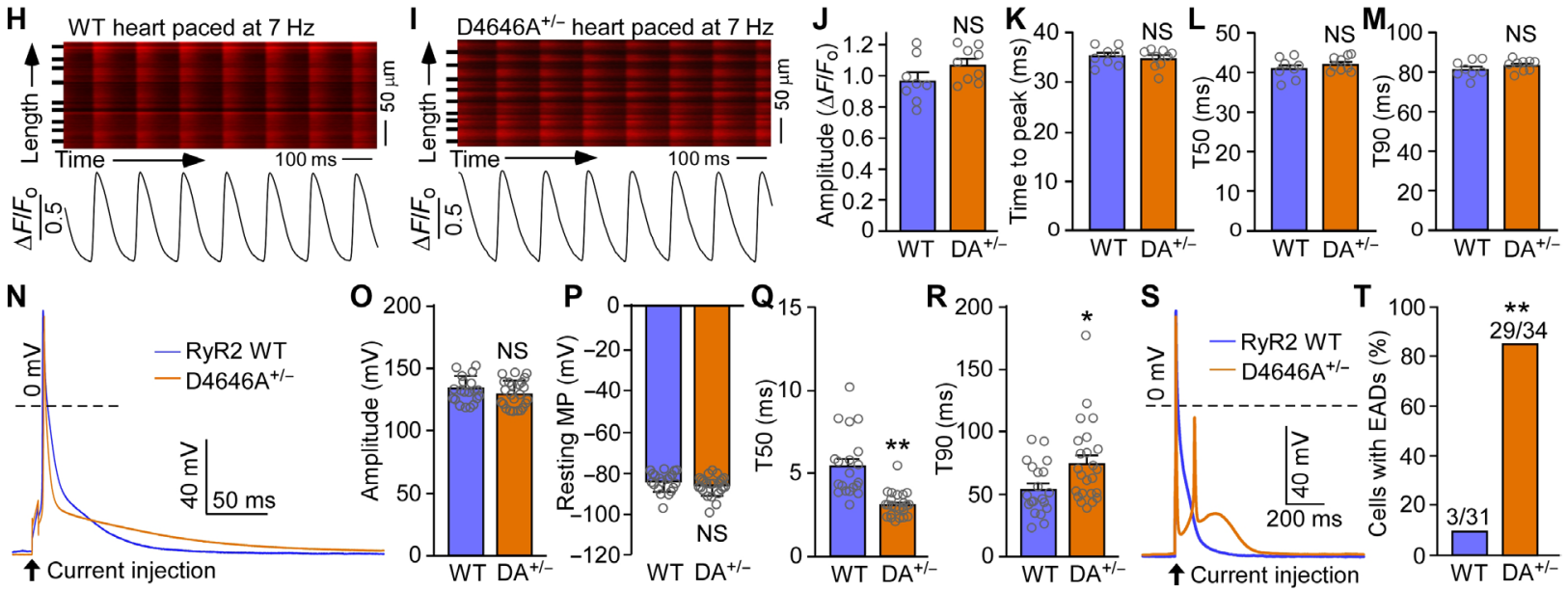

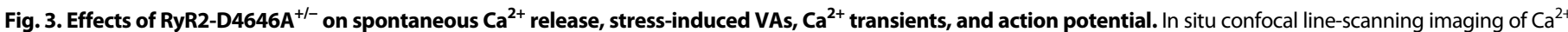

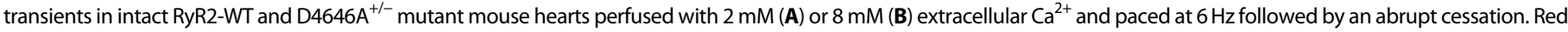

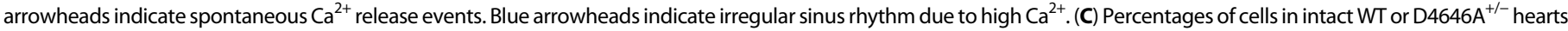

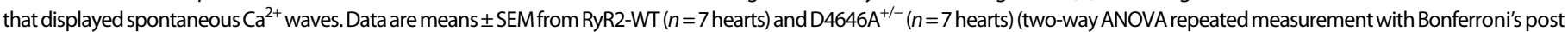

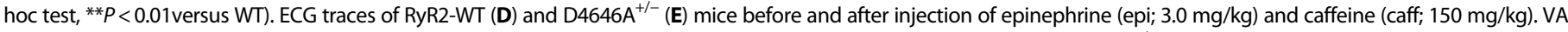

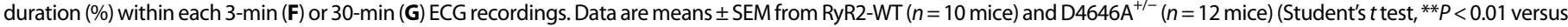

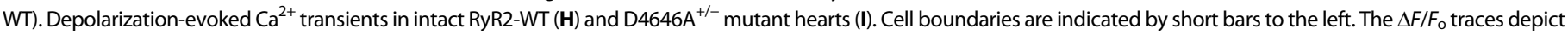

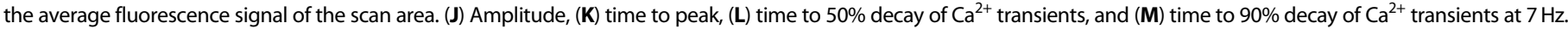

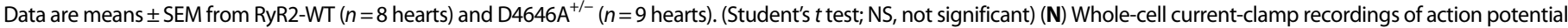

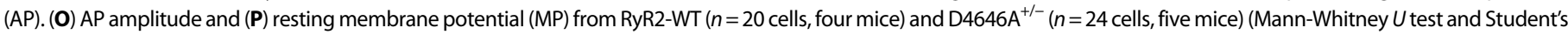

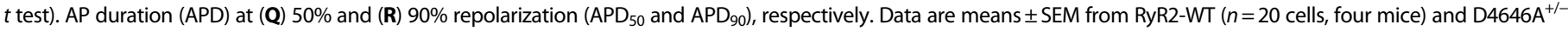

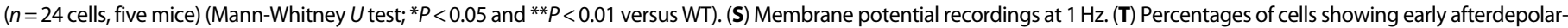
izations (EADs). Data are means \pm SEM from RyR2-WT ( $n=31$ cells, six mice) (chi-square test; ${ }^{* *} P<0.01$ versus WT) and D4646A ${ }^{+/-}(n=34$ cells, six mice).

RyR2-CRDS would otherwise be concealed before the onset of life-threatening VAs. Hence, this diagnostic tool may distinguish dangerous RyR2 LOF mutations from the benign background genomic noise.

\section{Quinidine and flecainide suppress VA in CRDS model mice}

We used the LBLPS protocol and the $\mathrm{D} 4646 \mathrm{~A}^{+/-}$mouse model to explore potential medical therapies for CRDS. Our electrophysiological studies revealed that the RyR2 D4646 $\mathrm{A}^{+/-}$mutation results in 

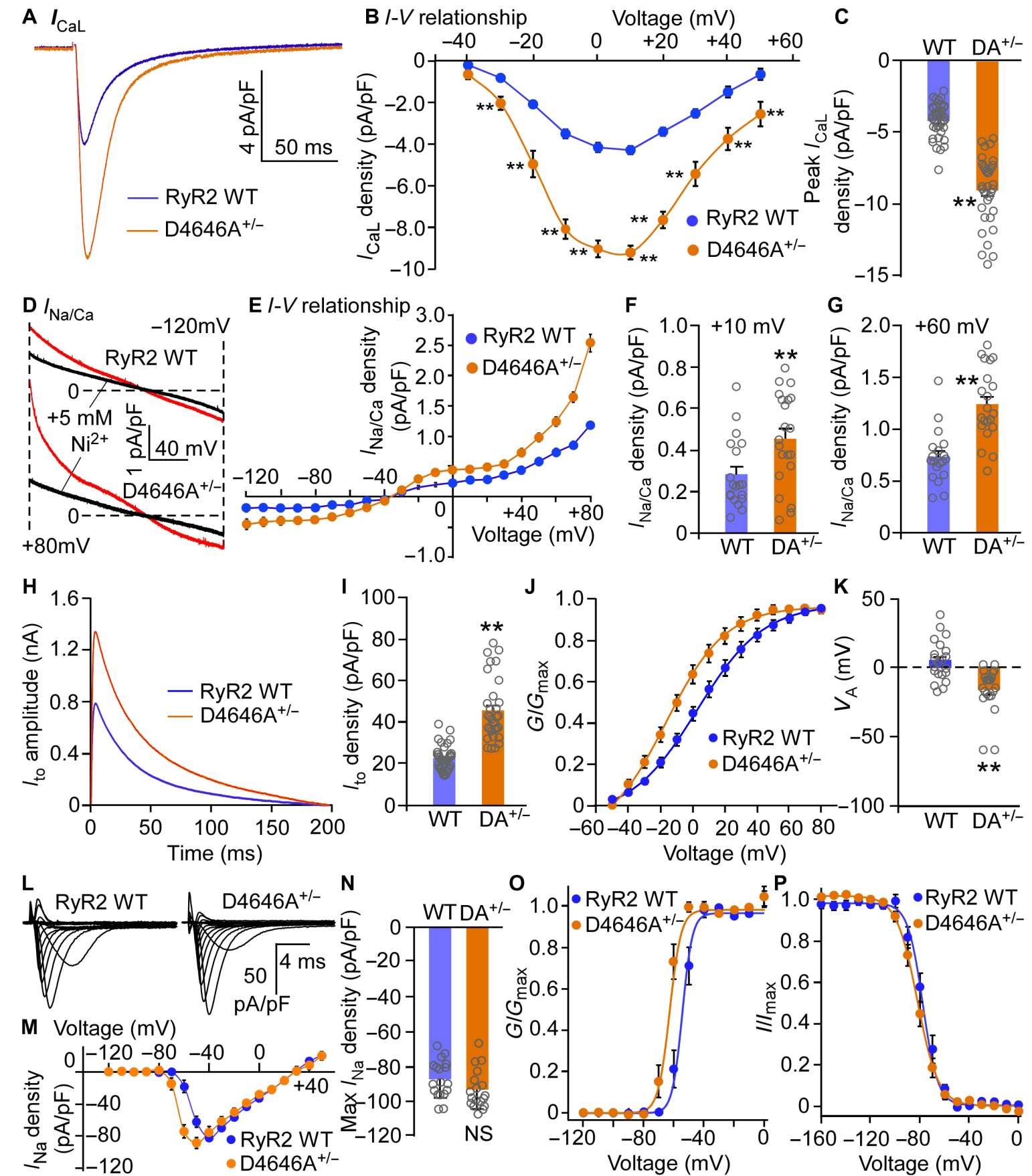

Fig. 4. Effects of RyR2-D4646A ${ }^{+/-}$on $\mathrm{Ca}^{2+}, \mathrm{K}^{+}$, and $\mathrm{Na}^{+}$currents in murine ventricular myocytes. (A) Whole-cell voltage-clamp recording of the $\mathrm{L}-$ type $\mathrm{Ca}{ }^{2+}$ current $\left(I_{\text {CaL }}\right)$. (B) Current-voltage relationship (I-V curve) of $I_{\text {CaL }}$ (C) Peak $I_{\text {CaL }}$ densities at $+10 \mathrm{mV}$. Data are means \pm SEM from RyR2-WT ( $n=39$ cells, five mice) and D4646A ${ }^{+/-}$ $\left(n=35\right.$ cells, six mice). (D) The Na/Ca exchange current $\left(I_{\mathrm{NCX}}\right)$ before and after addition of $5 \mathrm{mM} \mathrm{Ni}{ }^{2+}$. (E) $I-V$ relationship of $I_{\mathrm{NCX}} I_{\mathrm{NCX}}$ densities at $+10 \mathrm{mV}(\mathbf{F})$ and at $+60 \mathrm{mV}$ (G). Data are means \pm SEM from RyR2-WT ( $n=19$ cells, four mice) and D4646 $\mathrm{A}^{+/-}$( $n=21$ cells, four mice). (H) Whole-cell voltage-clamp recording of the transient outward $\mathrm{K}^{+}$current $\left(I_{\text {to }}\right)$. (I) Peak $I_{\text {to }}$ densities. Data are means \pm SEM from RyR2-WT ( $n=38$ cells, four mice) and D4646A ${ }^{+/-}(n=33$ cells, five mice). (J) Voltage dependence of steadystate activation of $I_{\text {to }}\left(G\right.$ for $\mathrm{K}^{+}$conductance). (K) Half-activated voltage $\left(V_{\mathrm{A}}\right)$ of $I_{\text {to. }}$. Data are means $\pm S E M$ from RyR2-WT $\left(n=21\right.$ cells, four mice) and D4646A ${ }^{+/-}(n=20$ cells, five mice). (L) Whole-cell voltage-clamp recording of the sodium current $\left(I_{\mathrm{Na}}\right.$ ). (M) $I-V$ relationships of $I_{\mathrm{Na}}$. (N) Maximum $/$ Na density from RyR2-WT ( $n=18$ cells, four mice) and D4646A ${ }^{+/-}\left(n=18\right.$ cells, four mice). Voltage dependence of steady-state activation ( $\mathbf{O}$ ) and inactivation (P) of $I_{\text {Na. }}$. Data are means $\pm S E M$ from RyR2-WT ( $n=18$ cells, four mice) and D4646A ${ }^{+/-}$( $n=18$ cells, four mice) (Mann-Whitney $U$ test and Student's $t$ test; ${ }^{* *} P<0.01$ versus WT).

substantial electrophysiological remodeling, including increased L-type $\mathrm{Ca}^{2+}$ channel $\left(I_{\mathrm{CaL}}\right)$ and transient outward $\mathrm{K}^{+}\left(I_{\mathrm{to}}\right)$ currents, and a hyperpolarization shift in the voltage-dependent activation of the $\mathrm{Na}^{+}$channel (Fig. 4). Thus, suppressing these channels in
RyR2-D4646A ${ }^{+/-}$mouse hearts may limit the VA risk associated with CRDS. Quinidine sulfate and flecainide have multiple inhibitory actions on both outward and inward currents $\left(I_{\mathrm{Na}}, I_{\mathrm{CaL}}\right.$, and $\left.I_{\mathrm{to}}\right)(25-29)$. Pretreatment of the $\mathrm{D} 4646 \mathrm{~A}^{+/-}$mutant mice with 
A RyR2-D4646A ${ }^{+/-}$mutant mice

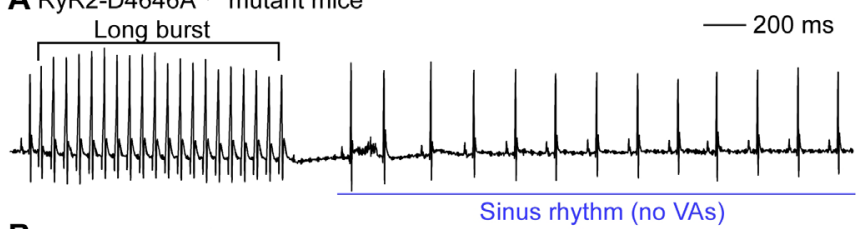

B RyR2-D4646A ${ }^{+/-}$mutant mice

Long burst + long pause

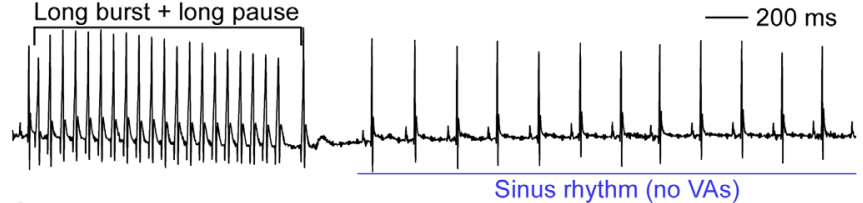

C $_{\text {RyR2-D4646A }}{ }^{+/-}$mutant mice

S1S2 stimulation

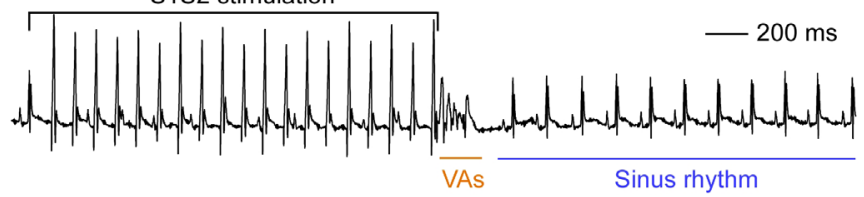

D

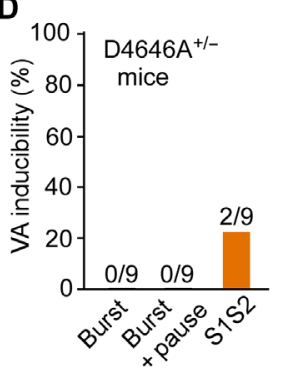

E

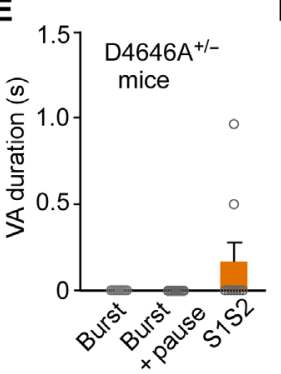

$\mathbf{F}$

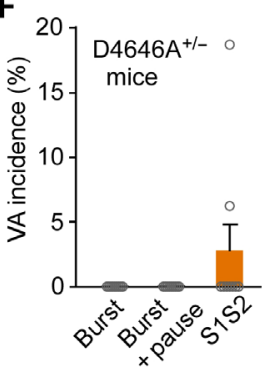

G RyR2 WT mice

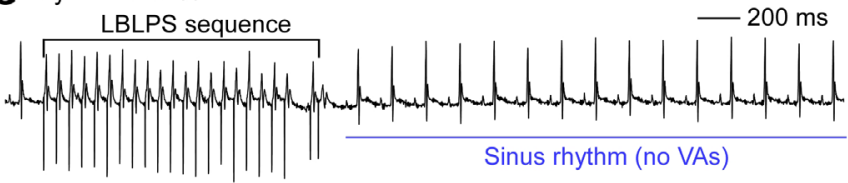

H RyR2-D4646A ${ }^{+/-}$LOF mice

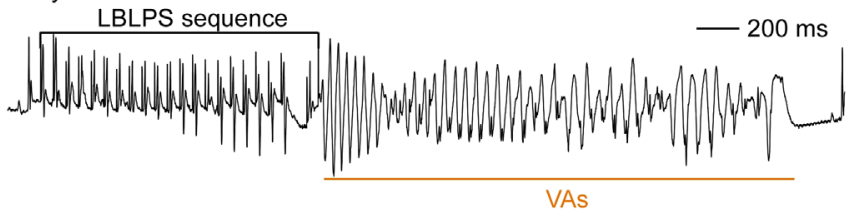

I RyR2-R4496C (-/- GOF mice

LBLPS sequence

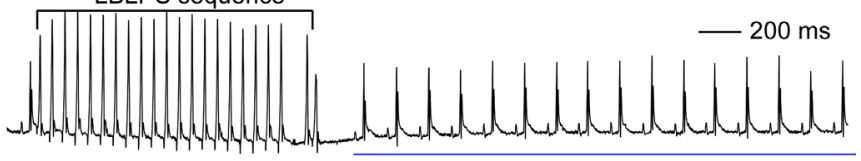

Sinus rhythm (no VAs)
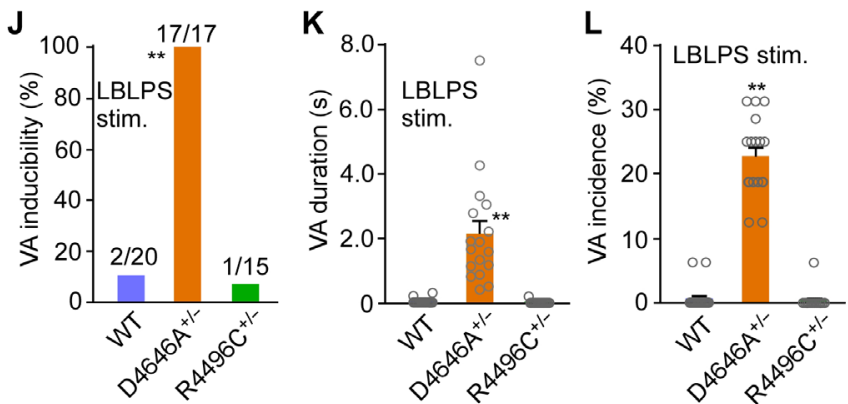

O A RyR2-T4196I ${ }^{+/-}$mutant carrier $2 \mathrm{~s}$

M N N ARyR2-13995V $\mathrm{V}^{+/-}$mutant carrier

ICD in a D4112 $\mathrm{N}^{+/-}$

mutant carrier

LBLPS sequence

$2 \mathrm{~s}$
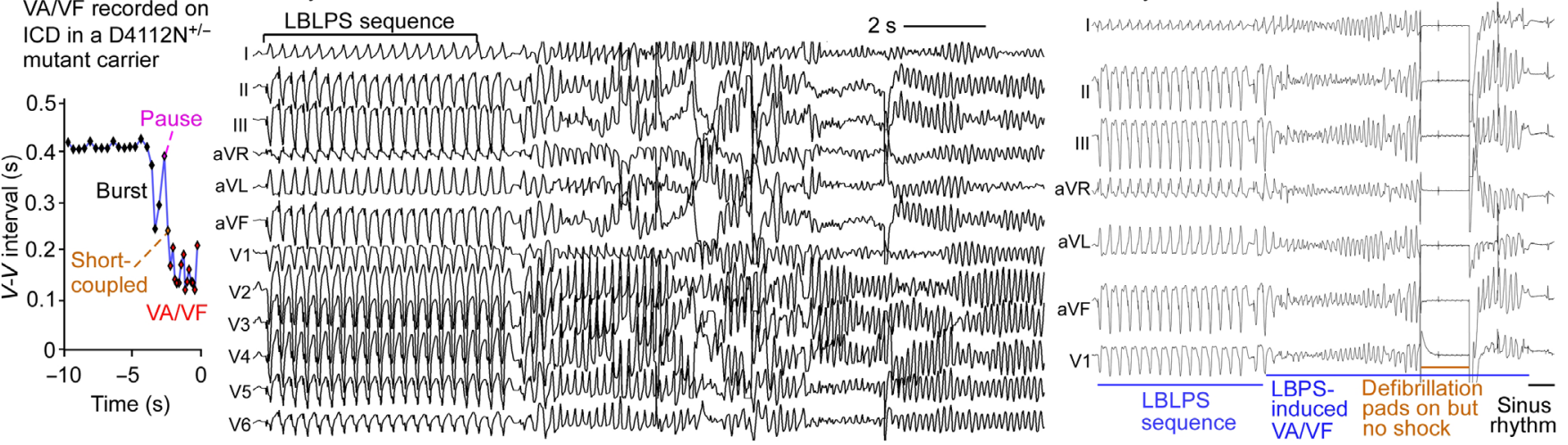

I MARM

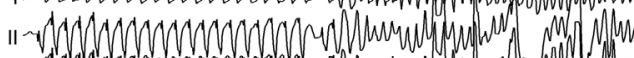

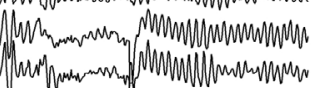

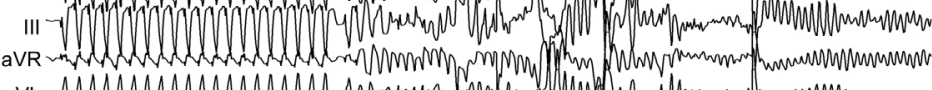

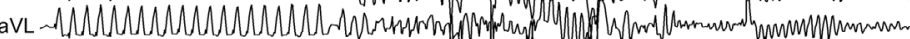

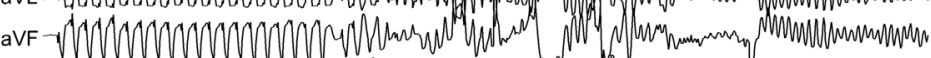
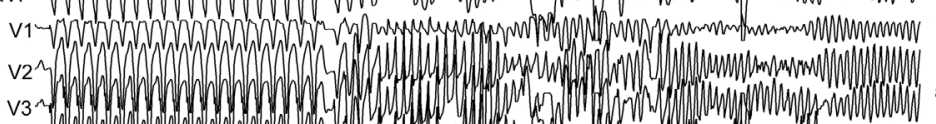

Mp.
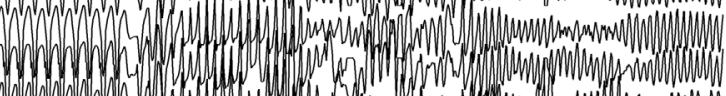

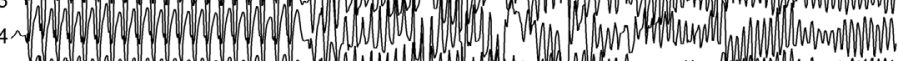

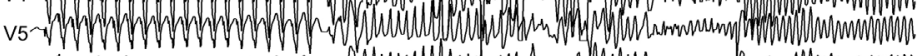

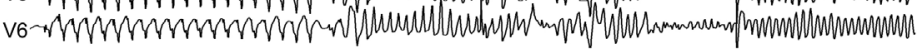

Fig. 5. VA induction in RyR2-WT and mutant mice and in humans with RyR2 LOF mutations by programed electrical stimulation. (A) ECG trace after a long burst (20 beats, 60-ms interval). (B) ECG trace after a long burst followed by a long pause (122 ms) and a stimulus. (C) ECG trace after a basal pacing (S1, 20 beats, 100-ms interval) followed by a short-coupled extra-stimulus (S2). The S1S2 interval was progressively reduced from 78 to $18 \mathrm{~ms}$ with 4-ms steps. (D) VA inducibility, (E) averaged VA duration, and (F) VA incidence in RyR2-D4646A ${ }^{+/-}$mice paced by different stimulation protocols. Data are means \pm SEM from D4646A ${ }^{+/-}\left(n=9\right.$ mice). ECG traces recorded in RyR2-WT (G), D4646A ${ }^{+/-}$ (H), and R4496C $\mathrm{C}^{+/}$(I) mice after the long-burst, long-pause, and short-coupled (LBLPS) stimulation protocol. (J) VA inducibility, (K) averaged VA duration, and (L) VA incidence in RyR2-WT, D4646A ${ }^{+/}$, and R4496C ${ }^{+/-}$mice after the LBLPS stimulation. Data are means \pm SEM from RyR2-WT ( $n=20$ mice), D4646A ${ }^{+-}\left(n=17\right.$ mice), and R4496C ${ }^{+/-}(n=15$ mice) (chi-square test; Kruskal-Wallis test with Dunn-Bonferroni post hoc test, ${ }^{* *} P<0.01$ versus WT). (M) $V$ - $V$ intervals recorded on ICD in a RyR2-D4112 ${ }^{+/-}$mutant carrier before and after the

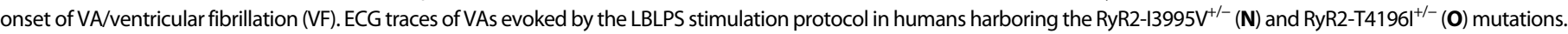

quinidine sulfate $(40 \mathrm{mg} / \mathrm{kg}$ per day for 6 days) or flecainide $(20 \mathrm{mg} /$ $\mathrm{kg}$ per day for 6 days) markedly reduced the duration and incidence of LBLPS-evoked polymorphic VAs (Fig. 6). This indicates that pharmacological suppression of $I_{\mathrm{to}}, I_{\mathrm{Na}}$, and $I_{\mathrm{CaL}}$ (combined) may serve as a potentially viable anti-CRDS therapeutic strategy.

\section{Large $\mathrm{Ca}^{2+}$ transient amplitude due to enhanced $\mathrm{SR} \mathrm{Ca}^{2+}$ load triggers LBLPS-evoked VAs in D4646 ${ }^{+/-}$hearts}

We next used the LBLPS protocol to better understand the trigger mechanism of VA in hearts harboring a CRDS RyR2 mutation. To this end, we assessed intracellular $\mathrm{Ca}^{2+}$ dynamics in 
A RyR2-D4646A ${ }^{+/-}$mice $\left(\mathrm{H}_{2} \mathrm{O}\right.$ control)

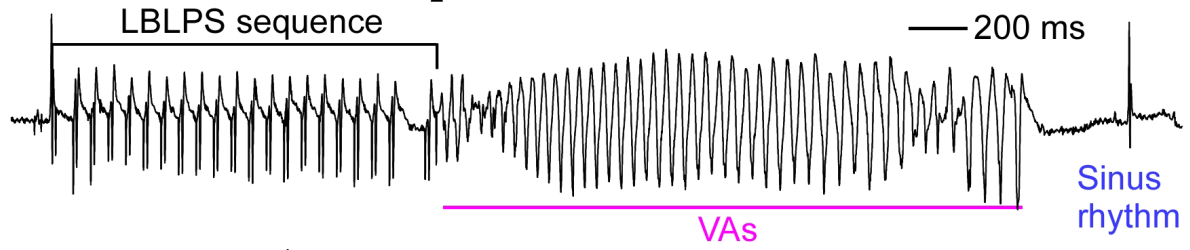

B RyR2-D4646A ${ }^{+/-}$mice (treated with quinidine sulfate, $10 \mathrm{mg} / \mathrm{kg}$ )

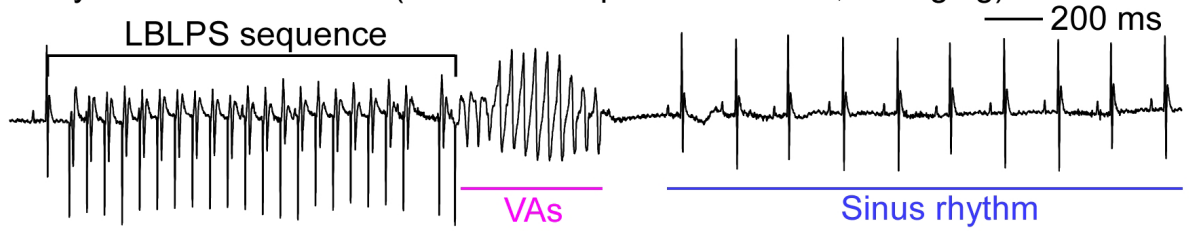

C RyR2-D4646A ${ }^{+/-}$mice (treated with quinidine sulfate, $40 \mathrm{mg} / \mathrm{kg}$ )

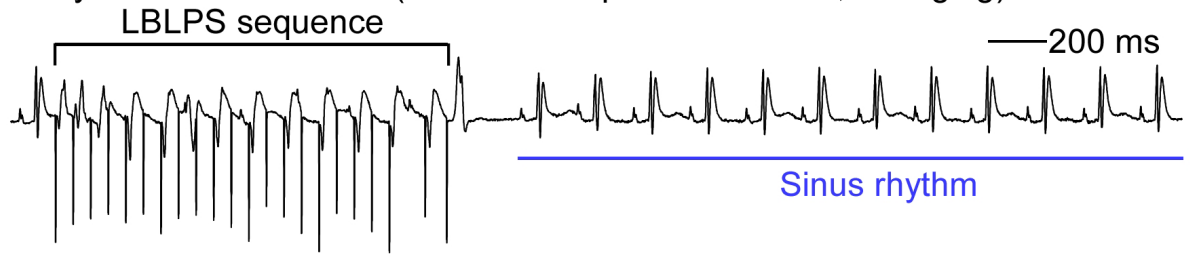

D RyR2-D4646A ${ }^{+/-}$mice (treated with flecainide, $4 \mathrm{mg} / \mathrm{kg}$ )

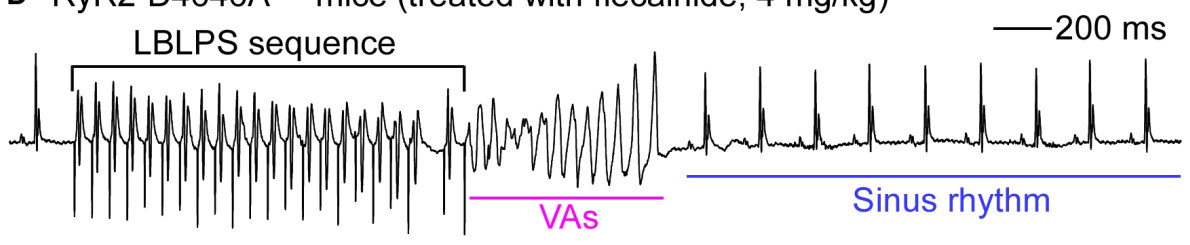

E RyR2-D4646A ${ }^{+/-}$mice (treated with flecainide, $20 \mathrm{mg} / \mathrm{kg}$ )
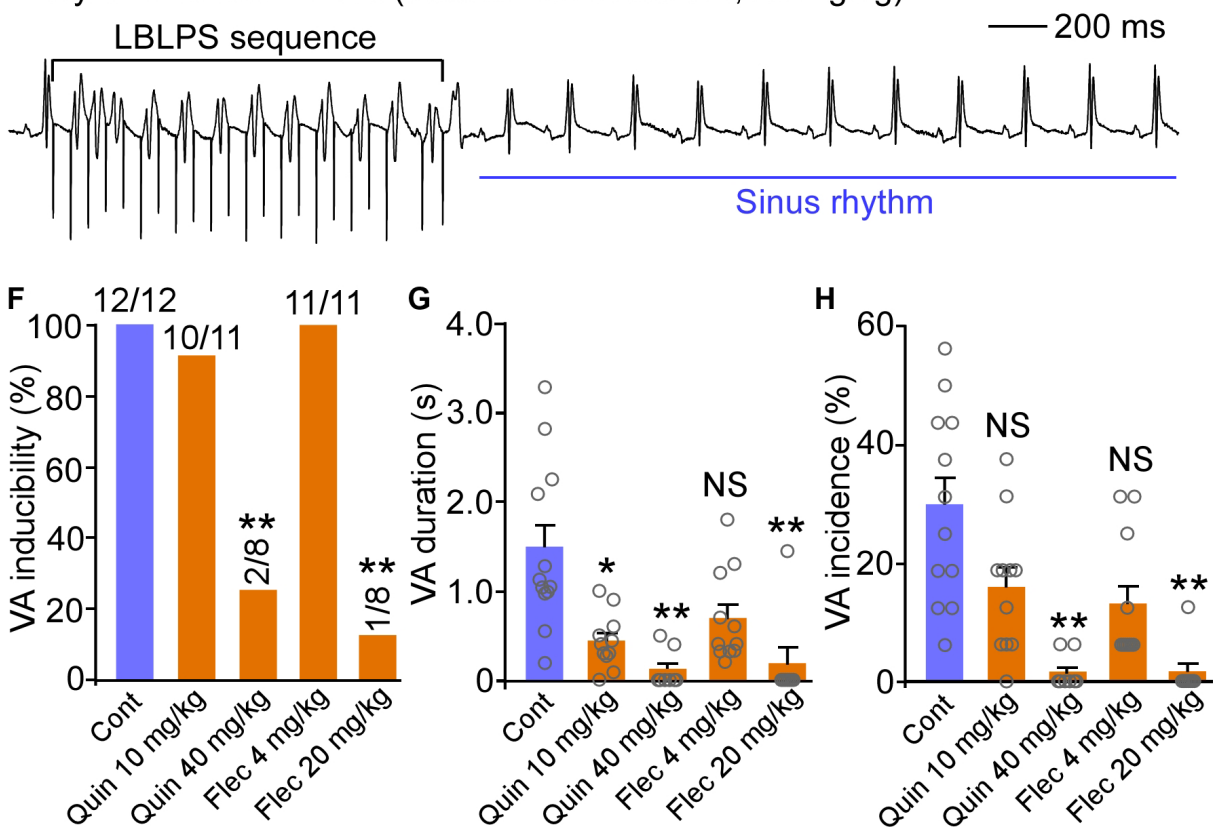

Fig. 6. Quinidine and flecainide suppress LBLPS-evoked VA in D4646A ${ }^{+/-}$mutant mice. ECG traces recorded in $\mathrm{D}_{4646 \mathrm{~A}^{+/-}}$mice after the LBLPS stimulation after injection of vehicle $\left(\mathrm{H}_{2} \mathrm{O}\right.$, control) (A), quinidine sulfate (10 or $40 \mathrm{mg} / \mathrm{kg}$ per day for 6 days, ip) (B and C) or flecainide ( 4 or $20 \mathrm{mg} / \mathrm{kg}$ per day for 6 days, ip) (D and E) with VA inducibility $(\mathbf{F})$, averaged VA duration $(\mathbf{G})$, and VA incidence $(\mathbf{H})$ shown. Data are means \pm SEM from D4646A ${ }^{+/-}$mutant mice ( $n=12$ mice for control, $n=11$ mice for $10 \mathrm{mg} / \mathrm{kg}$ per day of quinidine, $n=8$ mice for $40 \mathrm{mg} / \mathrm{kg}$ per day of quinidine, $n=11$ mice for $4 \mathrm{mg} / \mathrm{kg}$ per day of flecainide, and $n=8$ for $20 \mathrm{mg} / \mathrm{kg}$ per day of flecainide) (chi-square test; KruskalWallis test with Dunn-Bonferroni post hoc test, ${ }^{*} P<0.05$ and ${ }^{* *} P<0.01$ versus control). cardiomyocytes in intact hearts from RyR2-WT, RyR2-D4646A ${ }^{+/}$(CRDS), or RyR2-R4496C ${ }^{+/-}$(CPVT) mutant mice using in situ confocal $\mathrm{Ca}^{2+}$ imaging. LBLPS induced VA-like irregular $\mathrm{Ca}^{2+}$ transients in the CRDS RyR2-D4646A $\mathrm{A}^{+/-}$ hearts but not in CPVT RyR2-R4496C ${ }^{+/-}$ or WT hearts (Fig. 7, A to F), indicating that the RyR2-CRDS hearts are uniquely prone to LBLPS-induced VA-like $\mathrm{Ca}^{2+}$ transient irregularity. During the burst phase of LBLPS stimulation, the amplitude of $\mathrm{Ca}^{2+}$ transients (normalized to that at $6 \mathrm{~Hz}$ ) was reduced in CRDS RyR2-D4646A ${ }^{+/-}$hearts but not in CPVT RyR2-R4496C $\mathrm{C}^{+-}$hearts (Fig. 7, A to C and $\mathrm{G}$ to I). This is likely due to the prolonged SR $\mathrm{Ca}^{2+}$ release refractoriness present in the RyR2-D4646A ${ }^{+/-}$CRDS model heart.

There was no difference in either the amplitude or decay time of the $\mathrm{Ca}^{2+}$ transients at $6 \mathrm{~Hz}$ in WT, CRDS (D4646A $\left.{ }^{+/-}\right)$, and CPVT (R4496C $\mathrm{C}^{+/-}$) hearts (Fig. 7, $\mathrm{G}$ and $\mathrm{H}$, and fig. S11, A to C). However, the amplitude of $\mathrm{Ca}^{2+}$ transients after the long pause (normalized to that at $6 \mathrm{~Hz}$ ) was increased in CRDS (D4646A $\mathrm{A}^{+/-}$) hearts but not in CPVT (R4496C $\mathrm{C}^{+-}$) hearts (Fig. 7, A to C and J). This increase in $\mathrm{Ca}^{2+}$ transient amplitude after the long pause in CRDS (D4646 $\mathrm{A}^{+/-}$) hearts could reflect an elevation in $\mathrm{SR} \mathrm{Ca}^{2+}$ load. To directly test this prediction, we measured the SR Ca ${ }^{2+}$ content after the burst and long pause. The SR $\mathrm{Ca}^{2+}$ content in

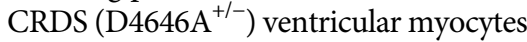
was elevated compared to WT, whereas the SR Ca ${ }^{2+}$ load in CPVT $\left(\mathrm{R}_{4496 \mathrm{C}^{+/}}\right)$ myocytes was reduced (fig. S11, D to $\mathrm{G})$. The elevated SR $\mathrm{Ca}^{2+}$ load in the $\mathrm{D}_{4} 646 \mathrm{~A}^{+/-}$ventricular myocytes is unlikely to be related to phospholamban (PLB), because phosphorylation of PLB was decreased in the $\mathrm{D} 4646 \mathrm{~A}^{+/-}$hearts compared to WT, although the total protein expression of PLB was unchanged (fig. S10).

In light of the enhanced $I_{\mathrm{NCX}}$ and increased EAD propensity in CRDS $\left(\mathrm{D} 4646 \mathrm{~A}^{+/-}\right)$hearts, the increased $\mathrm{Ca}^{2+}$ transient amplitude after the long pause is highly likely to induce EADs. Thus, the burst-pause-induced increase in the $\mathrm{Ca}^{2+}$ transient amplitude may act as a trigger for LBLPS-induced VAs via an EAD-mediated reentrant mechanism. To test this possibility, we performed high-resolution optical mapping to 
A RyR2 WT heart Long pause Short-
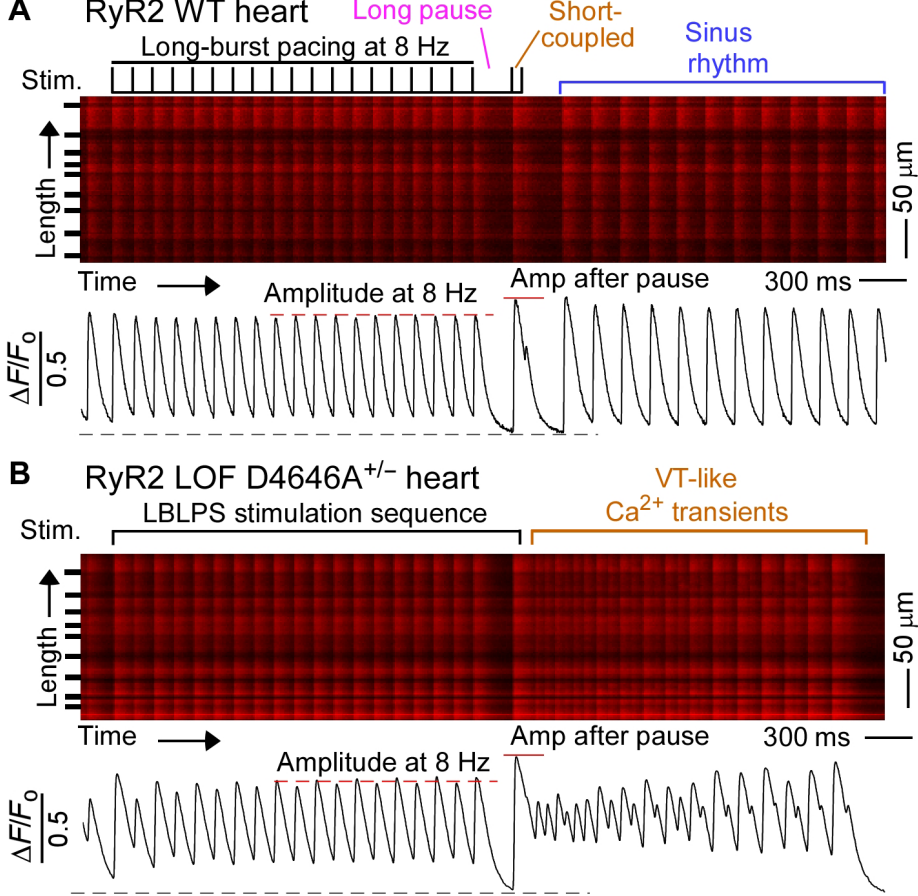

C RyR2 GOF R4496C ${ }^{+/-}$heart
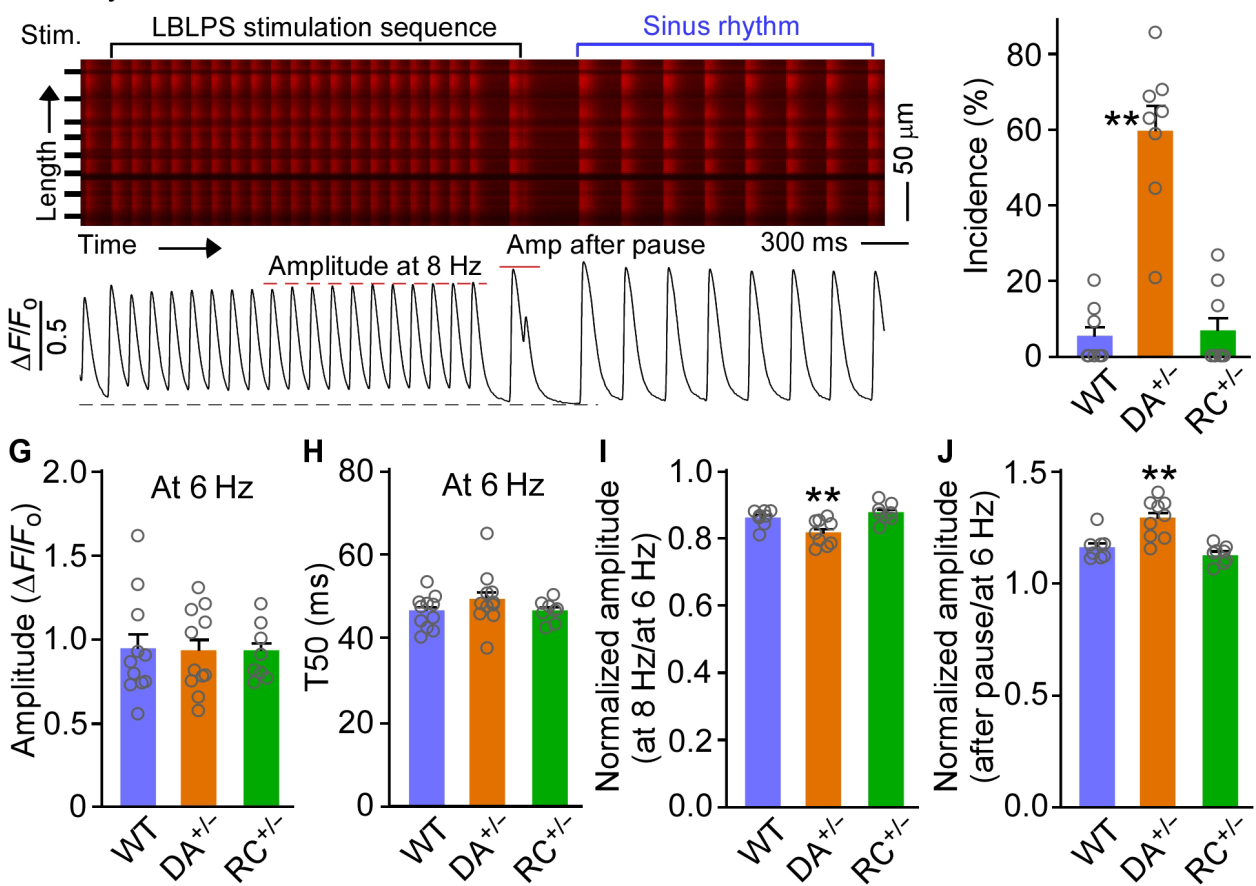

Fig. 7. LBLPS stimulation induces VA-like irregular $\mathrm{Ca}^{2+}$ transients in intact $\mathrm{D4646A}^{+/-}$LOF hearts but not in WT or R4496 $\mathrm{C}^{+/}$GOF mouse hearts. $\mathrm{Ca}^{2+}$ transients in intact RyR2-WT (A), D4646A $\mathrm{A}^{+/-}$(B), and R4496C $\mathrm{C}^{+-}$(C) mutant hearts after the LBLPS stimulation. (D) Inducibility, (E) average duration, and (F) incidence of VA-like irregular $\mathrm{Ca}^{2+}$ transients evoked by LBLPS. Data are means \pm SEM from RyR2-WT ( $n=8$ hearts), D4646A ${ }^{+/-}$( $n=8$ hearts), and $\mathrm{R}_{4496 \mathrm{C}^{+/-}}$( $n=9$ hearts) (chi-square test; Kruskal-Wallis test with Dunn-Bonferroni post hoc test, ${ }^{*} P<0.01$ versus WT). (G) Amplitude and (H) time to $50 \%$ decay of $\mathrm{Ca}^{2+}$ transients in intact RyR2-WT, DA ${ }^{+/}$, and $\mathrm{RC}^{+/-}$hearts paced at $6 \mathrm{~Hz}$. Data are means \pm SEM from RyR2-WT ( $n=11$ hearts), $\mathrm{DA}^{+/-}\left(n=11\right.$ hearts), and $\mathrm{RC}^{+/-}(n=8$ hearts). Amplitudes of $\mathrm{Ca}^{2+}$ transients at $8 \mathrm{~Hz}$ during the long burst $(\mathbf{I})$ and after the long pause $(\mathrm{J})$ of the LBLPS protocol, normalized to the transient amplitude at $6 \mathrm{~Hz}$, in RyR2-WT, $\mathrm{DA}^{+/-}$, and $\mathrm{RC}^{+/-}$hearts. Data are means \pm SEM from RyR2-WT ( $n=8$ hearts), $\mathrm{DA}^{+/-}\left(n=9\right.$ hearts), and RC $C^{+/-}\left(n=8\right.$ hearts) (chi-square test; one-way ANOVA with Dunnett's post hoc test, ${ }^{* *} P<0.01$ versus WT). measure patterns of conduction in isolated Langendorff-perfused RyR2-WT and RyR2-D4646A $\mathrm{A}^{+/-}$mutant hearts. As expected, all WT hearts showed activations near the apex of the heart that conducted uniformly through the right and left ventricles with no arrhythmias after LBLPS stimulation. In contrast, all RyR2-D4646A ${ }^{+/-}$mutant hearts displayed a reentrant pattern of conduction after LBLPS stimulation (fig. S12, A to C). We also observed APD alternans in all RyR2-D4646A ${ }^{+-}$mutant hearts but not in WT hearts during the long-burst (9-Hz) component of the LBLPS stimulation sequence (fig. S12D). Given the close link between electrical rotors and reentrant activity, our optical mapping data support the notion that RyR2-CRDS enhances the propensity for reentrant arrhythmias.

\section{DISCUSSION}

Multiple RyR2 GOF mutations are known to cause CPVT, which is routinely diagnosed clinically using the EST $(4,6-8,10,11)$. In the setting of unexplained cardiac arrests, reports have emerged in the literature that have identified patients having RyR2 mutations with negative EST (12, 15-17). The pathological significance of these RyR2 mutations is often unclear given that $3 \%$ of the general population has a rare RyR2 variant $(18,19)$. In this context, it may be very difficult to distinguish whether this genetic result is a benign, incidental finding or the underlying culprit responsible for the unexplained cardiac arrests. Recent observations have suggested that pathogenic RyR2 LOF mutations may account for these findings $(12,15-17)$; however, reports to date have been limited to single patients or small families, precluding a definitive link between genotype and phenotype. Unfortunately, this has resulted in patients being left with unclear diagnoses, which has often precluded optimal management of both probands and potentially vulnerable family members. Here, our systematic clinical, genetic, functional, and linkage analyses establish that RyR2 LOF mutations cause a previously unknown disease entity associated with life-threatening VAs, which we refer to as RyR2-CRDS. The clinical importance of establishing RyR2CRDS as a previously unknown arrhythmia 
syndrome extends beyond just providing an explanation to currently unexplained SCD/aSCD cases. Proper diagnosis of RyR2CRDS not only allows for optimal management of the proband but may also help to save the lives of family members also having life-threatening RyR2 LOF mutations.

RyR2 GOF mutations cause CPVT by promoting abnormal diastolic spontaneous SR $\mathrm{Ca}^{2+}$ release $(4,21,22,24,30-32)$, which drives DADs and thus increases the risk of triggered activities and VAs $(4,33-35)$. In stark contrast, we show that CRDS RyR2-D4646 $\mathrm{A}^{+/-}$LOF mutation abolished abnormal diastolic spontaneous SR Ca ${ }^{2+}$ release in intact mouse hearts, completely protecting them against DAD-driven VAs. Thus, the arrhythmogenic mechanism associated with RyR2-CRDS is different from that of CPVT. The CRDS RyR2-D4646A ${ }^{+/-}$mutation causes a substantial electrophysiological remodeling in the heart, increasing $I_{\mathrm{to}}, I_{\mathrm{CaL}}$, and $I_{\mathrm{NCX}}$. This alters AP waveform (i.e., shortens $\mathrm{APD}_{50}$ and lengthens $\mathrm{APD}_{90}$ ) and increases the propensity for arrhythmogenic EADs in the CRDS hearts. Thus, RyR2 LOF may cause VAs via an EADmediated mechanism, similar to that proposed for the RyR2-A4860 G ${ }^{+/-}$ LOF mutation (14).

Until now, there have been no diagnostic tests for RyR2CRDS. However, there is a critical need for developing a diagnostic test capable of unmasking the dangerous RyR2-CRDS phenotype. This would enable classification of a rare RyR2 variant as potentially dangerous or benign without requiring in vitro functional testing. Using our CRDS RyR2-D4646A $\mathrm{A}^{+-}$mouse model, we established a three-component stimulation sequence, termed LBLPS. Although sharing some common features, this LBLPS sequence is slightly different from the previously described "abrupt short-to-long cycle length change" protocol (36), in the relatively long-burst pacing period before the long pause. We tested LBLPS on WT, CPVT (RyR2-R4496C $\mathrm{C}^{+/-}$), and CRDS (RyR2-D4646A $\mathrm{A}^{+/-}$) mice and demonstrated that LBLPS is an effective and specific test for RyR2CRDS, because it induced VAs only in CRDS (RyR2-D4646A ${ }^{+/-}$)

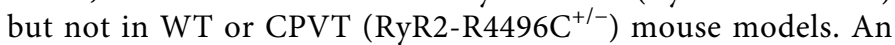
electrocardiogram (ECG) pattern similar to the LBLPS protocol was captured just before VA onset in a patient with RyR2-CRDS, suggesting that LBLPS may be able to trigger VAs in patients with RyR2-CRDS. In two patients with RyR2-CRDS, the LBLPS protocol successfully induced polymorphic VAs. Thus, our LBLPS stimulation protocol represents a promising means to potentially diagnose and assess SCD risk in patients with RyR2-CRDS.

The LBLPS sequence not only provides a potential CRDS diagnostic tool but also provides a means to explore the triggering mechanism underlying RyR2 LOF-associated VAs. Our studies show that LBLPS stimulation results in a substantial increase in SR $\mathrm{Ca}^{2+}$ load after a relatively long period of burst pacing and the long pause, likely as a result of increased $\mathrm{Ca}^{2+}$ influx $\left(I_{\mathrm{CaL}}\right)$ and diminished SR Ca ${ }^{2+}$ leak due to RyR2 LOF. Elevated SR $\mathrm{Ca}^{2+}$ load has also been observed in the RyR2-A4860G ${ }^{+/-}$and RyR2-E4872Q ${ }^{+/-}$LOF mutant mouse models $(14,37)$. Thus, increased SR Ca ${ }^{2+}$ load may be a common consequence of RyR2 LOF mutations during rapid pacing. The consequence is an aberrantly large $\mathrm{Ca}^{2+}$ transient during the post-pause beat in the CRDS (RyR2-D4646 $\mathrm{A}^{+/}$) hearts. Given the enhanced $I_{\mathrm{NCX}}$ and increased EAD propensity in these hearts, this large $\mathrm{Ca}^{2+}$ transient would increase the likelihood that a short-coupled extra-stimulus will induce EADs and EAD-mediated arrhythmias. In other words, the aberrantly large $\mathrm{Ca}^{2+}$ transient during the post-pause beat after rapid pacing is likely what triggers VAs in the EAD-prone CRDS RyR2-D4646A ${ }^{+/-}$heart by the LBLPS sequence. Thus, RyR2 $\mathrm{Ca}^{2+}$ release deficiency likely increases the propensity for producing both the trigger and the substrate for EAD-mediated reentrant arrhythmia. In support of this view, we observed reentrant-related electrical rotor activities after LBLPS stimulation in the RyR2-D4646A ${ }^{+/-}$mutant hearts but not in WT.

Given the broad electrophysiological remodeling occurring in the RyR2 LOF hearts, simply targeting one augmented current (e.g., $I_{\mathrm{Na}}$, $I_{\mathrm{to}}, I_{\mathrm{CaL}}$, or $I_{\mathrm{NCX}}$ ) may not be effective or may even be harmful, as suppressing one of these enhanced currents may perturb the newly adapted balance between depolarization and repolarization in the RyR2 LOF hearts. To minimize potential perturbation to the newly adapted balance, one could simultaneously target both the outward and inward currents. In support of this idea, we found that both quinidine and flecainide (inhibitors of $I_{\mathrm{to}}, I_{\mathrm{CaL}}$, and $\left.I_{\mathrm{Na}}\right)(25,26)$ suppress LBLPSevoked VAs in CRDS (RyR2-D4646A $\mathrm{A}^{+/-}$) model hearts, suggesting that these agents may serve as effective anti-CRDS therapeutics.

The pathophysiological ramifications of RyR2 LOF mutations are heterogeneous. The RyR2-A4860G ${ }^{+/-}$mutation, for example, is associated with idiopathic ventricular fibrillation (12). The RyR2$\mathrm{A} 4860 \mathrm{G}^{+/-}$mutation reduces $\mathrm{Ca}^{2+}$ transient amplitude but has no effect on the amplitude of $I_{\mathrm{CaL}}(14)$. On the other hand, the RyR2-D4646A ${ }^{+/-}$mutation has no effect on the $\mathrm{Ca}^{2+}$ transient amplitude but markedly increases $I_{\mathrm{CaL}}$. The RyR2-I4855M $\mathrm{M}^{+/-}$mutation is associated not only with cardiac arrhythmias but also with left ventricular non-compaction (LVNC) (16). The RyR2-K4594R $\mathrm{R}^{+/-}$/

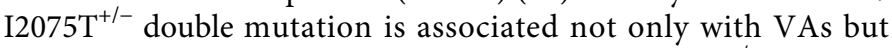
also with seizures (17). Furthermore, the RyR2-S4938 $\mathrm{F}^{+-}$mutation is associated with short-coupled variant of Torsade de Pointes (scTdP) (15). Therefore, not all RyR2 LOF mutations have the same pathogenic ramifications in the heart and affect function of other tissues. The resulting phenotypic heterogeneity is likely due to different degrees of cardiac remodeling and/or various degrees of pathogenic RyR2 dysfunction in other tissues. In this regard, our RyR2-D4646A ${ }^{+/-}$LOF mutant mouse model is unlikely to recapitulate all of the phenotypes observed with RyR2-CRDS in humans. Thus, CRDS ought to be considered as a multifaceted syndrome.

There are several limitations in the present study. It is unknown how RyR2 LOF results in increased $I_{\mathrm{to}}, I_{\mathrm{CaL}}$, and $I_{\mathrm{NCX}}$ with unchanged total protein expression. Further detailed and comprehensive investigations are required to assess potential changes in the expression of modulators and posttranslational modifications of these channels/exchangers. It is also unclear why patients with RyR2 LOF mutations have normal QT intervals despite that RyR2 LOF substantially altered the AP waveform in RyR2 LOF model mice. It is possible that the concomitant increases in both the outward $\mathrm{K}^{+}$current $\left(I_{\mathrm{to}}\right)$ and the inward $\mathrm{Ca}^{2+}$ current $\left(I_{\mathrm{CaL}}\right)$ may compensate for each other with respect to the overall APD. As a result, RyR2 LOF may have minimal impact on the QT interval. The low number of patients with RyR2-CRDS that we could recruit for testing is also a limitation. Further electrophysiological studies of more patients with CRDS will be needed to fully establish the CRDS specificity and sensitivity of the LBLPS protocol in humans.

In summary, this study establishes RyR2 LOF as causative for a previously unknown inherited arrhythmia syndrome that we have termed RyR2-CRDS. The RyR2-CRDS phenotype lacks catecholamine-induced ventricular ectopy, distinguishing it from CPVT. Unlike CPVT, ventricular ectopy in RyR2-CRDS arises 
secondary to marked electrophysiological remodeling that increases the heart's susceptibility to EAD-triggered VAs. Driven by the observation that RyR2-CRDS is effectively concealed before patients manifesting with potentially lethal events, we developed a ventricular pacing protocol that effectively unmasks the condition and may serve as a CRDS diagnostic test, although additional validation in humans will be required.

\section{MATERIALS AND METHODS Study design}

This study was designed to systematically determine whether RyR2 LOF is linked to a previously unknown disease entity characterized by SCD with negative EST, to understand the molecular mechanism by which RyR2 LOF causes SCD, and to develop diagnostic tools and treatments for patients with RyR2 LOF mutations. To achieve these objectives, we (i) performed clinical and genetic evaluations of families presenting with SCD or aSCD, negative EST, and RyR2 mutations; (ii) functionally characterized the identified RyR2 mutations; (iii) generated a mouse model harboring the RyR2 LOF mutation D4646A and assessed its impact on $\mathrm{Ca}^{2+}$ handling, electrophysiological and structural remodeling, and susceptibility to arrhythmias; (iv) developed a programmed stimulation protocol for the diagnosis of RyR2 LOF mutant carriers; and (v) identified potentially efficacious therapies for patients with RyR2 LOF mutations. All animal studies were approved by the Institutional Animal Care and Use Committees at the University of Calgary and were performed in accordance with U.S. National Institutes of Health guidelines. All animal experiments were performed blindly to genotype, and animals were assigned randomly to the experimental groups. Sample sizes for animal studies were calculated on the basis of previous studies and pilot studies. Sample sizes and $P$ values can be found in the figure legends. Individual subject-level data are reported in data file S1. The study was performed as part of protocols approved by the research ethics boards of all collaborating institutions that provided human clinical data. All human study participants provided informed consent for their clinical and genetic data to be used for research.

\section{Clinical and genetic evaluations of index cases and families}

To systematically establish whether LOF mutations in RyR2 are linked to a previously unknown entity of cardiac arrhythmia distinct from CPVT, we searched for RyR2 mutations that are associated with SCD or aSCD in the setting of negative EST and determined their impact on RyR2 channel function. Individuals who suffered an aborted cardiac arrest requiring resuscitation, SCD, or had documented polymorphic VT and were subsequently identified to have a rare RyR2 mutation [defined as an allele frequency of $<0.0001$ in the Genome Aggregation Database (gnomAD)] in the setting of EST that did not reveal evidence of prominent VAs during exertion consistent with a diagnosis of CPVT were eligible for inclusion into the study (18). All patients were required to have undergone a coronary assessment, either through coronary or computerized tomography coronary angiography, to exclude a myocardial ischemic culprit for their presentation and echocardiography to rule out structural heart disease. Family pedigrees were constructed for each index case. Cascade screening through both clinical phenotyping and genetic testing for the suspected pathogenic RyR2 mutation was performed in all accessible and consenting family members. Electrophysiology studies were performed in eligible affected patients using an LBLPS stimulation protocol. Full details of the electrophysiology studies are provided in the Supplementary Materials. There were no premature ventricular contractions (PVCs) observed during the testing, and QT intervals of our patients under all conditions tested were within the normal limit.

\section{Functional evaluation of RyR2 mutations}

The functional ramifications of RyR2 mutations associated with $\mathrm{SCD} / \mathrm{aSCD}$ were assessed by monitoring spontaneous or caffeineinduced $\mathrm{Ca}^{2+}$ release in HEK293 cells (American Type Culture Collection). Free cytosolic $\mathrm{Ca}^{2+}$ concentration in HEK293 cells was measured using the fluorescence $\mathrm{Ca}^{2+}$ indicator dye Fluo-3-AM or Fura-2/AM (Invitrogen), as previously described $(21,22,38)$.

\section{Generation and characterization of a mouse model of RyR2 $\mathrm{Ca}^{2+}$ release deficiency}

To understand the molecular and cellular mechanisms of RyR2CRDS, we generated a mouse model harboring one of the human RyR2 LOF mutations in patients, D4646A, using clustered regularly interspaced short palindromic repeats technology. Briefly, guide RNAs (5'-GGTCATTTCCCAATAACTACTGG-3') were designed and validated for the efficiency of targeting the ryr 2 gene in mice. A mixture of active guide RNA transcripts, single-stranded oligodeoxynucleotide donor, and Cas-9 mRNA was injected into the cytoplasm of C57BL/6 embryos. Newborn mice were screened for knock-in of the designed mutation by polymerase chain reactionbased restriction digest using primers $5^{\prime}$-ccaagccacagttcctaaacatt- $3^{\prime}$ (forward) and $5^{\prime}$-agagtgtggatttcttggatgct- $3^{\prime}$ (reverse) and the Hae III restriction enzyme. Founder mutant mice harboring the RyR2D4646A mutation were confirmed by DNA sequencing. All mice are maintained in the $129 \mathrm{E}$ background. Systolic and diastolic $\mathrm{Ca}^{2+}$ dynamics in ventricular myocytes in the Langendorff-perfused hearts were recorded using laser scanning confocal $\mathrm{Ca}^{2+}$ imaging as previously described $(39,40)$. Adult genetically engineered mice RyR2-D4646A (DA) (generated commercially by Applied StemCell) and RyR2-R4496C (RC) (41) and WT littermates of both sexes were used. All animal models were housed in the mouse facility of the Cumming School of Medicine, University of Calgary. Only heterozygous RyR2-D4646A ${ }^{+/-}$mutant mice were produced, suggesting that the homozygous $\mathrm{D}_{4646 \mathrm{~A}^{+/+}}$mutation is embryonic lethal. Functional RyR2s are tetrameric channels formed by four RyR2 monomers. The heterozygous D4646 $\mathrm{A}^{+/-}$mutant mice (harboring one WT allele and one D4646A mutant allele) will produce a mixture of homo- and hetero-tetrameric channels that contain the WT, D4646A mutant, or both WT/D4646A mutant monomers. Thus, the RyR2-D4646A mutation can exert its negative impact not only on the function of the D4646A homo-tetramers but also on the function of the WT monomer in the form of the WT/D4646A mutant hetero-tetrameric channels. Note that as in humans, the RyR2D $4646 \mathrm{~A}^{+/-}$LOF mutation affects both male and female mice.

\section{Stress-induced VAs in mice}

The susceptibility of RyR2-WT and mutant mice to stress-induced VAs was assessed using ECG recordings. Mice were lightly anesthetized with isoflurane vapor $(0.5$ to $1 \%)$ and $95 \% \mathrm{O}_{2}$. Anesthetized mice were placed on a heating pad $\left(37^{\circ} \mathrm{C}\right)$, and needle electrodes were inserted subcutaneously into the right upper limb and left lower abdomen for lead II ECG recording (BIOPAC MP System, Goleta, $\mathrm{CA})$. Baseline ECG was recorded for 5 to $10 \mathrm{~min}$. The mice were 
subjected to intraperitoneal injection of a mixture of epinephrine $(3.0 \mathrm{mg} / \mathrm{kg})$ and caffeine $(150 \mathrm{mg} / \mathrm{kg})$. Continuous ECG monitoring was performed for $30 \mathrm{~min}$ after the injection (41).

\section{Mouse intracardiac ECG recordings}

Intracardiac atrial, atrioventricular nodal, and ventricular electrograms were recorded simultaneously in anesthetized RyR2-WT and mutant mice using a 1.1-F octa-polar catheter (EPR-800, Millar Instruments, Houston, TX). To induce VAs, an LBLPS pacing protocol with a long burst ( 20 beats at a $60-\mathrm{ms}$ cycle length) followed by a long-coupled ventricular extra-stimulus (122 ms) and a subsequent short-coupled extra-stimulus was used. The short-coupled interval was progressively reduced from 78 to $18 \mathrm{~ms}$ in 4 -ms steps. VA was defined as four or more ventricular ectopic beats after pacing, VA inducibility (\%) as the proportion of animals that developed VAs per group, average VA duration as the summed duration of VA/total VA episodes for each group, and VA incidence (\%) as the total number of VA episodes/total numbers of pacing trials for each group.

\section{Statistical analysis}

All data shown are means \pm SEM, unless indicated otherwise. For normally distributed datasets, parametric tests were performed. For non-Gaussian-distributed datasets, nonparametric methods were used. With respect to nonparametric analyses, Mann-Whitney $U$ test (two-sided) was used for two groups; Kruskal-Wallis test with Dunn's post hoc test was used for three or more than three groups. With respect to parametric analyses, Student's $t$ test (two-sided) was used for two groups; one-way or two-way analysis of variance (ANOVA) followed by either Dunnett's or Bonferroni's post hoc test was used for three or more than three groups. The chi-square test was used to determine significant differences in frequencies between different groups. Sample sizes and $P$ values can be found in the figure legends. $P$ values smaller than 0.05 were considered statistically significant.

\section{SUPPLEMENTARY MATERIALS}

stm.sciencemag.org/cgi/content/full/13/579/eaba7287/DC1

Materials and Methods

Fig. S1. Pedigrees of families with non-CPVT-associated RyR2 mutations.

Fig. S2. Non-CPVT-associated RyR2 mutations suppress caffeine-induced $\mathrm{Ca}^{2+}$ release.

Fig. S3. Effects of mutations on the expression and function of RyR2.

Fig. S4. The D4646A mutation suppresses $\mathrm{Ca}^{2+}$ activation of single RyR2 channels in lipid bilayers.

Fig. S5. Generation and functional characterization of knock-in mouse model expressing the RyR2 mutation D4646A ${ }^{+/-}$.

Fig. S6. Effects of RyR2-D4646A ${ }^{+/-}$on caffeine-promoted spontaneous $\mathrm{Ca}^{2+}$ release and depolarization-induced $\mathrm{Ca}^{2+}$ transients.

Fig. S7. Effects of RyR2-D4646 $\mathrm{A}^{+/-}$on $\mathrm{Ca}^{2+}$ alternans and $\mathrm{Ca}^{2+}$ release refractoriness.

Fig. S8. Effects of RyR2-D4646A ${ }^{+/-}$on the $I_{\text {to }}$ current, cardiac structure and fibrosis, L-type Ca ${ }^{2+}$ channel surface expression, and oxidative stress.

Fig. S9. Effects of RyR2-D4646A ${ }^{+/-}$on the expression of $\mathrm{Ca}^{2+}$ handling proteins, ion channels, and their associated proteins.

Fig. S10. Effects of RyR2-D4646 $\mathrm{A}^{+/-}$on the expression of $\mathrm{Ca}^{2+}$ handling protein, ion channel, phosphatases, and kinases.

Fig. S11. Effects of RyR2 mutations on $\mathrm{Ca}^{2+}$ transients and SR Ca ${ }^{2+}$ contents.

Fig. S12. Electrical conduction in the RyR2-WT and D4646A $\mathrm{A}^{+/-}$hearts.

Table S1. LOD scores for each family specified by $R Y R 2$ mutation.

Table S2. Echocardiographic parameters of RyR2-WT and D4646A ${ }^{+/-}$mutant mice.

Data file S1. Individual subject-level data.

References (42-54)

View/request a protocol for this paper from Bio-protocol.

\section{REFERENCES AND NOTES}

1. D. M. Bers, Cardiac excitation-contraction coupling. Nature 415, 198-205 (2002).

2. D. M. Bers, Calcium and cardiac rhythms: Physiological and pathophysiological. Circ. Res. 90, 14-17 (2002)

3. M. Fill, J. A. Copello, Ryanodine receptor calcium release channels. Physiol. Rev. 82, 893-922 (2002).

4. S. G. Priori, S. R. Chen, Inherited dysfunction of sarcoplasmic reticulum $\mathrm{Ca}^{2+}$ handling and arrhythmogenesis. Circ. Res. 108, 871-883 (2011).

5. S. G. Priori, C. Napolitano, N. Tiso, M. Memmi, G. Vignati, R. Bloise, V. V. Sorrentino, G. A. Danieli, Mutations in the Cardiac Ryanodine Receptor Gene ( $h R y R 2)$ underlie catecholaminergic polymorphic ventricular tachycardia. Circulation 103, 196-200 (2001).

6. C. Napolitano, S. G. Priori, Diagnosis and treatment of catecholaminergic polymorphic ventricular tachycardia. Heart Rhythm 4, 675-678 (2007).

7. A. Medeiros-Domingo, Z. A. Bhuiyan, D. J. Tester, N. Hofman, H. Bikker, J. P. van Tintelen, M. M. Mannens, A. A. Wilde, M. J. Ackerman, The RYR2-encoded ryanodine receptor/ calcium release channel in patients diagnosed previously with either catecholaminergic polymorphic ventricular tachycardia or genotype negative, exercise-induced long QT syndrome: A comprehensive open reading frame mutational analysis. J. Am. Coll. Cardiol. 54, 2065-2074 (2009).

8. K. V. Lieve, C. van der Werf, A. A. Wilde, Catecholaminergic polymorphic ventricular tachycardia. Circ. J. 80, 1285-1291 (2016).

9. M. Hayashi, I. Denjoy, F. Extramiana, A. Maltret, N. R. Buisson, J.-M. Lupoglazoff, D. Klug, M. Hayashi, S. Takatsuki, E. Villain, J. Kamblock, A. Messali, P. Guicheney, J. Lunardi, A. Leenhardt, Incidence and risk factors of arrhythmic events in catecholaminergic polymorphic ventricular tachycardia. Circulation 119, 2426-2434 (2009).

10. T. M. Roston, J. M. Vinocur, K. R. Maginot, S. Mohammed, J. C. Salerno, S. P. Etheridge, M. Cohen, R. M. Hamilton, A. Pflaumer, R. J. Kanter, J. E. Potts, M. J. La Page, K. K. Collins, R. A. Gebauer, J. D. Temple, A. S. Batra, C. Erickson, M. Miszczak-Knecht, P. Kubuš, Y. Bar-Cohen, M. Kantoch, V. C. Thomas, G. Hessling, C. Anderson, M.-L. Young, M. C. Ortega, Y. R. Lau, C. L. Johnsrude, A. Fournier, P. J. Kannankeril, S. Sanatani, Catecholaminergic polymorphic ventricular tachycardia in children: Analysis of therapeutic strategies and outcomes from an international multicenter registry. Circ. Arrhythm. Electrophysiol. 8, 633-642 (2015).

11. T. M. Roston, S. Sanatani, S. R. Chen, Suppression-of-function mutations in the cardiac ryanodine receptor: Emerging evidence for a novel arrhythmia syndrome? Heart Rhythm 14, 108-109 (2017)

12. S. G. Priori, C. Napolitano, M. Memmi, B. Colombi, F. Drago, M. Gasparini, L. De Simone, F. Coltorti, R. Bloise, R. Keegan, F. E. S. Cruz Filho, G. Vignati, A. Benatar, A. De Logu, Clinical and molecular characterization of patients with catecholaminergic polymorphic ventricular tachycardia. Circulation 106, 69-74 (2002).

13. D. Jiang, W. Chen, R. Wang, L. Zhang, S. R. W. Chen, Loss of luminal Ca ${ }^{2+}$ activation in the cardiac ryanodine receptor is associated with ventricular fibrillation and sudden death. Proc. Natl. Acad. Sci. U.S.A. 104, 18309-18314 (2007).

14. Y.-T. Zhao, C. R. Valdivia, G. B. Gurrola, P. P. Powers, B. C. Willis, R. L. Moss, J. Jalife, H. H. Valdivia, Arrhythmogenesis in a catecholaminergic polymorphic ventricular tachycardia mutation that depresses ryanodine receptor function. Proc. Natl. Acad. Sci. U.S.A. 112, E1669-E1677 (2015).

15. Y. Fujii, H. Itoh, S. Ohno, T. Murayama, N. Kurebayashi, H. Aoki, M. Blancard, Y. Nakagawa, S. Yamamoto, Y. Matsui, M. Ichikawa, K. Sonoda, T. Ozawa, K. Ohkubo, I. Watanabe, P. Guicheney, M. Horie, A type 2 ryanodine receptor variant associated with reduced $\mathrm{Ca}^{2+}$ release and short-coupled torsades de pointes ventricular arrhythmia. Heart Rhythm 14, 98-107 (2017).

16. T. M. Roston, W. Guo, A. D. Krahn, R. Wang, F. Van Petegem, S. Sanatani, S. R. W. Chen, A. Lehman, A novel RYR2 loss-of-function mutation (I4855M) is associated with left ventricular non-compaction and atypical catecholaminergic polymorphic ventricular tachycardia. J. Electrocardiol. 50, 227-233 (2017).

17. C. Paech, R. A. Gebauer, J. Karstedt, C. Marschall, A. Bollmann, D. Husser, Ryanodine receptor mutations presenting as idiopathic ventricular fibrillation: A report on two novel familial compound mutations, c.6224T $>C$ and c.13781 A $>G$, with the clinical presentation of idiopathic ventricular fibrillation. Pediatr. Cardiol. 35, 1437-1441 (2014).

18. M. Lek, K. J. Karczewski, E. V. Minikel, K. E. Samocha, E. Banks, T. Fennell, A. H. O'Donnell-Luria, J. S. Ware, A. J. Hill, B. B. Cummings, T. Tukiainen, D. P. Birnbaum, J. A. Kosmicki, L. E. Duncan, K. Estrada, F. Zhao, J. Zou, E. Pierce-Hoffman, J. Berghout, D. N. Cooper, N. Deflaux, M. De Pristo, R. Do, J. Flannick, M. Fromer, L. Gauthier, J. Goldstein, N. Gupta, D. Howrigan, A. Kiezun, M. I. Kurki, A. L. Moonshine, P. Natarajan, L. Orozco, G. M. Peloso, R. Poplin, M. A. Rivas, V. Ruano-Rubio, S. A. Rose, D. M. Ruderfer, K. Shakir, P. D. Stenson, C. Stevens, B. P. Thomas, G. Tiao, M. T. Tusie-Luna, B. Weisburd, H.-H. Won, D. Yu, D. M. Altshuler, D. Ardissino, M. Boehnke, J. Danesh, S. Donnelly, R. Elosua, J. C. Florez, S. B. Gabriel, G. Getz, S. J. Glatt, C. M. Hultman, S. Kathiresan, M. Laakso, S. M. Carroll, M. I. McCarthy, D. M. Govern, R. M. Pherson, B. M. Neale, A. Palotie, S. M. Purcell, D. Saleheen, J. M. Scharf, P. Sklar, P. F. Sullivan, J. Tuomilehto, M. T. Tsuang, H. C. Watkins, J. G. Wilson, M. J. Daly, 
D. G. MacArthur; Exome Aggregation Consortium, Analysis of protein-coding genetic variation in 60,706 humans. Nature 536, 285-291 (2016).

19. J. D. Kapplinger, K. N. Pundi, N. B. Larson, T. E. Callis, D. J. Tester, H. Bikker, A. A. M. Wilde, M. J. Ackerman, Yield of the RYR2 genetic test in suspected catecholaminergic polymorphic ventricular tachycardia and implications for test interpretation. Circ. Genom. Precis. Med. 11, e001424 (2018).

20. J. Ott, A computer program for linkage analysis of general human pedigrees. Am. J. Hum. Genet. 28, 528-529 (1976).

21. D. Jiang, B. Xiao, D. Yang, R. Wang, P. Choi, L. Zhang, H. Cheng, S. R. W. Chen, RyR2 mutations linked to ventricular tachycardia and sudden death reduce the threshold for store-overload-induced $\mathrm{Ca}^{2+}$ release (SOICR). Proc. Natl. Acad. Sci. U.S.A. 101, 13062-13067 (2004).

22. D. Jiang, R. Wang, B. Xiao, H. Kong, D. J. Hunt, P. Choi, L. Zhang, S. R. W. Chen, Enhanced store overload-induced $\mathrm{Ca}^{2+}$ release and channel sensitivity to luminal $\mathrm{Ca}^{2+}$ activation are common defects of RyR2 mutations linked to ventricular tachycardia and sudden death. Circ. Res. 97, 1173-1181 (2005).

23. M. Cerrone, B. Colombi, M. Santoro, M. R. di Barletta, M. Scelsi, L. Villani, C. Napolitano, S. G. Priori, Bidirectional ventricular tachycardia and fibrillation elicited in a knock-in mouse model carrier of a mutation in the cardiac ryanodine receptor. Circ. Res. 96, e77-e82 (2005).

24. N. Liu, B. Colombi, M. Memmi, S. Zissimopoulos, N. Rizzi, S. Negri, M. Imbriani, C. Napolitano, F. A. Lai, S. G. Priori, Arrhythmogenesis in catecholaminergic polymorphic ventricular tachycardia: Insights from a RyR2 R4496C knock-in mouse model. Circ. Res. 99 292-298 (2006).

25. Y. Imaizumi, W. R. Giles, Quinidine-induced inhibition of transient outward current in cardiac muscle. Am. J. Physiol. 253, H704-H708 (1987).

26. J. J. Salata, J. A. Wasserstrom, Effects of quinidine on action potentials and ionic currents in isolated canine ventricular myocytes. Circ. Res. 62, 324-337 (1988).

27. F. Scamps, A. Undrovinas, G. Vassort, Inhibition of ICa in single frog cardiac cells by quinidine, flecainide, ethmozin, and ethacizin. Am. J. Physiol. 256, C549-C559 (1989).

28. Z. Wang, B. Fermini, S. Nattel, Effects of flecainide, quinidine, and 4-aminopyridine on transient outward and ultrarapid delayed rectifier currents in human atrial myocytes. J. Pharmacol. Exp. Ther. 272, 184-196 (1995).

29. H. Watanabe, N. Chopra, D. Laver, H. S. Hwang, S. S. Davies, D. E. Roach, H. J. Duff, D. M. Roden, A. A. M. Wilde, B. C. Knollmann, Flecainide prevents catecholaminergic polymorphic ventricular tachycardia in mice and humans. Nat. Med. 15, 380-383 (2009).

30. P. J. Kannankeril, B. M. Mitchell, S. A. Goonasekera, M. G. Chelu, W. Zhang, S. Sood D. L. Kearney, C. I. Danila, M. De Biasi, X. H. T. Wehrens, R. G. Pautler, D. M. Roden, G. E. Taffet, R. T. Dirksen, M. E. Anderson, S. L. Hamilton, Mice with the R176Q cardiac ryanodine receptor mutation exhibit catecholamine-induced ventricular tachycardia and cardiomyopathy. Proc. Natl. Acad. Sci. U.S.A. 103, 12179-12184 (2006).

31. P. P. Jones, D. Jiang, J. Bolstad, D. J. Hunt, L. Zhang, N. Demaurex, S. R. Chen, Endoplasmic reticulum $\mathrm{Ca}^{2+}$ measurements reveal that the cardiac ryanodine receptor mutations linked to cardiac arrhythmia and sudden death alter the threshold for store-overload-induced $\mathrm{Ca}^{2+}$ release. Biochem. J. 412, 171-178 (2008).

32. S. Sedej, F. R. Heinzel, S. Walther, N. Dybkova, P. Wakula, J. Groborz, P. Gronau, L. S. Maier, M. A. Vos, F. A. Lai, C. Napolitano, S. G. Priori, J. Kockskämper, B. Pieske, $\mathrm{Na}^{+}$-dependent SR $\mathrm{Ca}^{2+}$ overload induces arrhythmogenic events in mouse cardiomyocytes with a human CPVT mutation. Cardiovasc. Res. 87, 50-59 (2010).

33. E. G. Lakatta, Functional implications of spontaneous sarcoplasmic reticulum $\mathrm{Ca}^{2+}$ release in the heart. Cardiovasc. Res. 26, 193-214 (1992).

34. E. Marban, S. W. Robinson, W. G. Wier, Mechanisms of arrhythmogenic delayed and early afterdepolarizations in ferret ventricular muscle. J. Clin. Invest. 78, 1185-1192 (1986).

35. C. H. Orchard, D. A. Eisner, D. G. Allen, Oscillations of intracellular $\mathrm{Ca}^{2+}$ in mammalian cardiac muscle. Nature 304, 735-738 (1983).

36. S. Denker, M. Lehmann, R. Mahmud, C. Gilbert, M. Akhtar, Facilitation of ventricular tachycardia induction with abrupt changes in ventricular cycle length. Am. J. Cardiol. 53, 508-515 (1984).

37. W. Chen, R. Wang, B. Chen, X. Zhong, H. Kong, Y. Bai, Q. Zhou, C. Xie, J. Zhang, A. Guo, X. Tian, P. P. Jones, M. L. O'Mara, Y. Liu, T. Mi, L. Zhang, J. Bolstad, L. Semeniuk, H. Cheng, J. Zhang, J. Chen, D. P. Tieleman, A. M. Gillis, H. J. Duff, M. Fill, L.-S. Song, S. R. W. Chen, The ryanodine receptor store-sensing gate controls $\mathrm{Ca}^{2+}$ waves and $\mathrm{Ca}^{2+}$-triggered arrhythmias. Nat. Med. 20, 184-192 (2014).

38. P. Li, S. R. Chen, Molecular basis of $\mathrm{ca}^{2}+$ activation of the mouse cardiac $\mathrm{ca}^{2}+$ release channel (ryanodine receptor). J. Gen. Physiol. 118, 33-44 (2001).

39. B. Chen, A. Guo, Z. Gao, S. Wei, Y.-P. Xie, S. R. W. Chen, M. E. Anderson, L.-S. Song, In situ confocal imaging in intact heart reveals stress-induced $\mathrm{Ca}^{2+}$ release variability in a murine catecholaminergic polymorphic ventricular tachycardia model of type 2 ryanodine receptor $^{\text {R4496C+/- }}$ mutation. Circ. Arrhythm. Electrophysiol. 5, 841-849 (2012).

40. Y. Bai, P. P. Jones, J. Guo, X. Zhong, R. B. Clark, Q. Zhou, R. Wang, A. Vallmitjana, R. Benitez, L. Hove-Madsen, L. Semeniuk, A. Guo, L.-S. Song, H. J. Duff, S. R. W. Chen, Phospholamban knockout breaks arrhythmogenic $\mathrm{Ca}^{2+}$ waves and suppresses catecholaminergic polymorphic ventricular tachycardia in mice. Circ. Res. 113 517-526 (2013)

41. Q. Zhou, J. Xiao, D. Jiang, R. Wang, K. Vembaiyan, A. Wang, C. D. Smith, C. Xie, W. Chen, J. Zhang, X. Tian, P. P. Jones, X. Zhong, A. Guo, H. Chen, L. Zhang, W. Zhu, D. Yang, X. Li, J. Chen, A. M. Gillis, H. J. Duff, H. Cheng, A. M. Feldman, L.-S. Song, M. Fill, T. G. Back, S. R. W. Chen, Carvedilol and its new analogs suppress arrhythmogenic store overload-induced $\mathrm{Ca}^{2+}$ release. Nat. Med. 17, 1003-1009 (2011).

42. S. N. Ho, H. D. Hunt, R. M. Horton, J. K. Pullen, L. R. Pease, Site-directed mutagenesis by overlap extension using the polymerase chain reaction. Gene 77, 51-59 (1989)

43. M. Zhao, P. Li, X. Li, L. Zhang, R. J. Winkfein, S. R. Chen, Molecular identification of the ryanodine receptor pore-forming segment. J. Biol. Chem. 274, 25971-25974 (1999).

44. U.K. Laemmli, Cleavage of structural proteins during the assembly of the head of bacteriophage T4 Nature 227, 680-685 (1970).

45. H. Towbin, T. Staehelin, J. Gordon, Electrophoretic transfer of proteins from polyacrylamide gels to nitrocellulose sheets: Procedure and some applications. Proc. Natl. Acad. Sci. U.S.A. 76, 4350-4354 (1979).

46. L. Wang, R. C. Myles, N. M. De Jesus, A. K. Ohlendorf, D. M. Bers, C. M. Ripplinger, Optical mapping of sarcoplasmic reticulum $\mathrm{Ca}^{2+}$ in the intact heart: Ryanodine receptor refractoriness during alternans and fibrillation. Circ. Res. 114, 1410-1421 (2014).

47. X. Zhong, B. Sun, A. Vallmitjana, T. Mi, W. Guo, M. Ni, R. Wang, A. Guo, H. J. Duff, A. M. Gillis, L.-S. Song, L. Hove-Madsen, R. Benitez, S. R. W. Chen, Suppression of ryanodine receptor function prolongs $\mathrm{Ca}^{2+}$ release refractoriness and promotes cardiac alternans in intact hearts. Biochem. J. 473, 3951-3964 (2016).

48. J. Xiao, X. Tian, P. P. Jones, J. Bolstad, H. Kong, R. Wang, L. Zhang, H. J. Duff, A. M. Gillis, S. Fleischer, M. Kotlikoff, J. A. Copello, S. R. W. Chen, Removal of FKBP12.6 does not alter the conductance and activation of the cardiac ryanodine receptor or the susceptibility to stress-induced ventricular arrhythmias. J. Biol. Chem. 282, 34828-34838 (2007).

49. A. E. Palmer, C. Jin, J. C. Reed, R. Y. Tsien, Bcl-2-mediated alterations in endoplasmic reticulum $\mathrm{Ca}^{2+}$ analyzed with an improved genetically encoded fluorescent sensor. Proc. Natl. Acad. Sci. U.S.A. 101, 17404-17409 (2004).

50. A. Fabiato, F. Fabiato, Calculator programs for computing the composition of the solutions containing multiple metals and ligands used for experiments in skinned muscle cells. J. Physiol. Paris 75, 463-505 (1979).

51. D. Lang, A. V. Glukhov, High-resolution optical mapping of the mouse sino-atrial node. J. Vis. Exp. , e54773 (2016)

52. I. Polina, H. J. Jansen, T. Li, M. Moghtadaei, L. J. Bohne, Y. Liu, P. Krishnaswamy, E. E. Egom, D. D. Belke, S. A. Rafferty, M. Ezeani, A. M. Gillis, R. A. Rose, Loss of insulin signaling may contribute to atrial fibrillation and atrial electrical remodeling in type 1 diabetes. Proc. Natl. Acad. Sci. U.S.A. 117, 7990-8000 (2020).

53. C. O'Shea, A. P. Holmes, T. Y. Yu, J. Winter, S. P. Wells, J. Correia, B. J. Boukens, J. R. De Groot, G. S. Chu, X. Li, G. A. Ng, P. Kirchhof, L. Fabritz, K. Rajpoot, D. Pavlovic ElectroMap: High-throughput open-source software for analysis and mapping of cardiac electrophysiology. Sci. Rep. 9, 1389 (2019).

54. E. Lander, L. Kruglyak, Genetic dissection of complex traits: Guidelines for interpreting and reporting linkage results. Nat. Genet. 11, 241-247 (1995).

Funding: This work was supported by research grants from the Canadian Institutes of Health Research (PJT-155940) to S.R.W.C. and (PJT 166105 and MOP 142486) to R.A.R.i the National Natural Science Foundation of China (81903611) to B.S.; the Spanish Ministry of Science Innovation and Universities SAF2017-88019-C3-R to L.H.-M. and R.B. and Marato-2015-20-30 to L.H.-M.; the Royal Netherlands Academy of Sciences (CVON 2012-10 PREDICT) to A.A.M.W.; the E-Rare Joint Transnational Call for Proposals 2015 "Improving Diagnosis and Treatment of Catecholaminergic Polymorphic Ventricular Tachycardia: Integrating Clinical and Basic Science" to S.R.W.C., S.S., and A.A.M.W.; the European Research Council Grant "EU-rhythmy" ERC-ADG-2014 (ID: 669387) to S.G.P.; the Novo Nordisk Foundation, Denmark (NNF180C0031258) to H.K.J.; and the NIH (R01HL057832) to M.F. B.S. and J.Y. are recipients of the Alberta Innovates-Health Solutions (AIHS) Fellowship Award. J.W. is a recipient of the Libin Cardiovascular Institute of Alberta and Cumming School of Medicine Postdoctoral Fellowship Award. X.Z. and W.G. are recipients of the AlHS Studentship Award. S.R.W.C. holds the Heart and Stroke Foundation Chair in Cardiovascular Research. Author contributions: B.S., J.Y., M.N., S.S., A.A.M.W., J.D.R., S.G.P., H.K.J., and S.R.W.C. designed 
the research. B.S., J.Y., M.N., J.W., X.Z., W.G., L.Z., and R.W. are responsible for molecular, cellular, electrophysiological, and/or whole animal studies; D.B. for echo analysis; Y.-X.C. for histological analysis; and K.V.V.L., A.K.B., T.M.R., I.B., J.A.K., J.C.v.A., and C.N. for clinical studies. B.S., J.Y., M.N., J.W., X.Z., W.G., L.Z., R.W., D.B., Y.-X.C., K.V.V.L., A.K.B., T.M.R., I.B., J.A.K., J.C.v.A., J.L., A.V., L.J.B., R.A.R., R.B., L.H.-M., C.N., R.A.H., S.S., A.A.M.W., J.D.R., S.G.P., H.K.J., and S.R.W.C. analyzed data. B.S., J.Y., M.N., R.W., K.V.V.L., T.M.R., J.L., A.V., L.J.B., R.A.R., R.B., L.H.-M., R.A.H., M.F., S.S., A.A.M.W., J.D.R., S.G.P., H.K.J., and S.R.W.C. wrote the paper. Competing interests: The authors declare that they have no competing interests. Data and materials availability: All data associated with this study are present in the paper or the Supplementary Materials.
Submitted 30 December 2019

Accepted 7 December 2020

Published 3 February 2021

$10.1126 /$ scitranslmed.aba7287

Citation: B. Sun, J. Yao, M. Ni, J. Wei, X. Zhong, W. Guo, L. Zhang, R. Wang, D. Belke, Y.-X. Chen, K. V. Lieve, A. K. Broendberg, T. M. Roston, I. Blankoff, J. A. Kammeraad, J. C. von Alvensleben, J. Lazarte, A. Vallmitjana, L. J. Bohne, R. A. Rose, R. Benitez, L. Hove-Madsen, C. Napolitano, R. A. Hegele, M. Fill, S. Sanatani, A. A. Wilde, J. D. Roberts, S. G. Priori, H. K. Jensen, S. R. W. Chen, Cardiac ryanodine receptor calcium release deficiency syndrome. Sci. Transl. Med. 13, eaba7287 (2021). 


\section{Science Translational Medicine}

\section{Cardiac ryanodine receptor calcium release deficiency syndrome}

Bo Sun, Jinjing Yao, Mingke Ni, Jinhong Wei, Xiaowei Zhong, Wenting Guo, Lin Zhang, Ruiwu Wang, Darrell Belke, Yong-Xiang Chen, Krystien V.V. Lieve, Anders K. Broendberg, Thomas M. Roston, Ivan Blankoff, Janneke A. Kammeraad, Johannes C. von Alvensleben, Julieta Lazarte, Alexander Vallmitjana, Loryn J. Bohne, Robert A. Rose, Raul Benitez, Leif Hove-Madsen, Carlo Napolitano, Robert A. Hegele, Michael Fill, Shubhayan Sanatani, Arthur A.M. Wilde, Jason D. Roberts, Silvia G. Priori, Henrik K. Jensen and S. R. Wayne Chen

Sci Transl Med 13, eaba7287.

DOI: 10.1126/scitransImed.aba7287

\section{Calcium and cardiac arrhythmia}

Calcium signaling couples cardiac electrical excitation and contraction and is tightly controlled within myocytes via the ryanodine receptor (RyR2). Sun et al. investigated loss-of-function mutations in RyR2 identified in families of individuals who experienced sudden cardiac death. RyR2 mutations were linked to cardiac arrhythmias due to prolonged calcium release refractoriness and electrophysiological and structural remodeling. The authors developed a cardiac stimulation protocol that induced ventricular arrhythmias in mice harboring a RyR2 mutation, and similar electrical patterns were seen in patients, which could be used to diagnose the RyR2 calcium release deficiency syndrome. Quinidine and flecainide could suppress arrhythmias in mice, suggesting possible treatments for this disease.

ARTICLE TOOLS

SUPPLEMENTARY MATERIALS

RELATED
CONTENT

REFERENCES

PERMISSIONS http://stm.sciencemag.org/content/13/579/eaba7287

http://stm.sciencemag.org/content/suppl/2021/02/01/13.579.eaba7287.DC1

http://stm.sciencemag.org/content/scitransmed/10/458/eaan0724.full http://stm.sciencemag.org/content/scitransmed/10/435/eaah5457.full http://stm.sciencemag.org/content/scitransmed/11/482/eaau8680.full

This article cites 53 articles, 27 of which you can access for free http://stm.sciencemag.org/content/13/579/eaba7287\#BIBL

http://www.sciencemag.org/help/reprints-and-permissions

Science Translational Medicine (ISSN 1946-6242) is published by the American Association for the Advancement of Science, 1200 New York Avenue NW, Washington, DC 20005. The title Science Translational Medicine is a registered trademark of AAAS.

Copyright (C 2021 The Authors, some rights reserved; exclusive licensee American Association for the Advancement of Science. No claim to original U.S. Government Works 